HEAT TRANSFER- ASIAN RESEARCH

Online ISSN:1523-1496; Publisher - Wiley.

Accepted May $28^{\text {th }} 2019$

\title{
Computational Unsteady Flow Analysis for Third-grade Fluid from an Isothermal Vertical Cylinder through a Darcian Porous Medium
}

\author{
Ashwini Hiremath ${ }^{1}$, G. Janardhana Reddy ${ }^{1,}{ }^{*}$ and O. Anwar Bég ${ }^{2}$ \\ ${ }^{1}$ Department of Mathematics, Central University of Karnataka, Kalaburagi, India \\ ${ }^{2}$ Fluid Mechanics, Aeronautical and Mechanical Engineering Department, School of Computing, \\ Science and Engineering, University of Salford, Manchester M54WT, UK \\ *Corresponding author Email: gjr@cuk.ac.in
}

\section{ABSTRACT:}

The present paper describes a mathematical model for free-convective laminar incompressible boundary layer flow of a third-grade fluid of the Reiner-Rivlin differential type, external to a uniformly heated semi-infinite vertical cylinder embedded in a two-dimensional porous medium. Assuming a homogenous-isotropic porous medium, simulation of bulk drag effects at low Reynolds number is conducted with the Darcy model. The resulting partial differential equation boundary value problem is normalized using suitable transformation variables. The highly non-linear time-dependent coupled conservation equations along with boundary conditions are solved computationally with an optimized Crank-Nicolson finite difference code. Validation with previous studies is included. The heat transport and skin friction coefficients are computed for different values of emerging non-dimensional parameters. Furthermore, steady-state and transient fluid-flow variables are shown graphically. An enhanced fluid velocity is observed for increased Darcy number and the reverse trend is computed for higher values of third-grade viscoelastic parameter. Also, the rate of heat transfer is observed to increase with greater Darcy number and a reduction in third-grade viscoelastic parameter. A key observation which is drawn from the present study is that for third-grade fluid the flow variables deviate significantly from a hot cylindrical wall as compared to a Newtonian fluid. The study is relevant to thermal polymer coating applications in aerospace materials processing.

KEYWORDS: Boussinesq's approximation; Third-grade Reiner-Rivlin fluid; Vertical cylinder; Finite difference method; Third-grade fluid parameter; Porous medium; Darcy number; Thermal polymer coating processing.

\section{Nomenclature}

$\mathrm{g}^{\prime} \quad$ acceleration due to gravity $\left(\mathrm{m} / \mathrm{s}^{2}\right)$

$k \quad$ thermal conductivity (W/m K) 


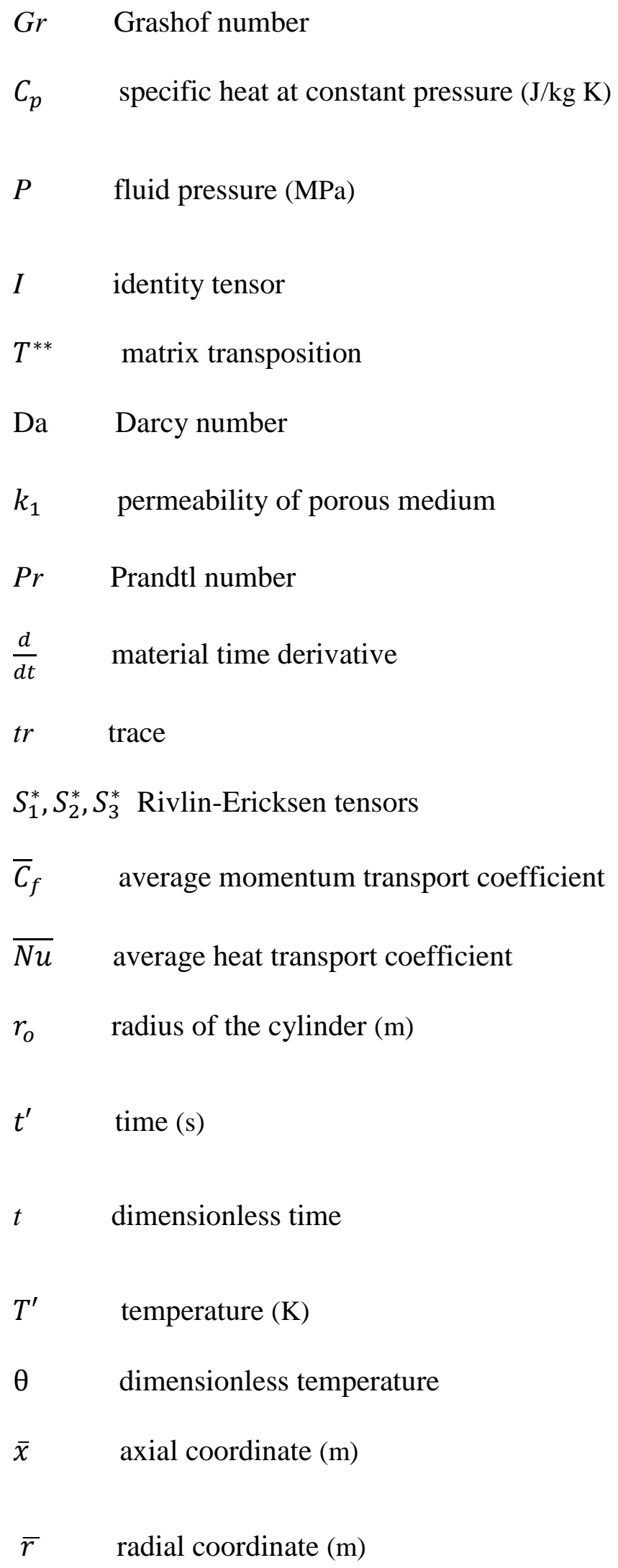


X dimensionless axial coordinate

$R$ dimensionless radial coordinate

$\bar{u}, \bar{v} \quad$ dimensional velocity coordinates $(\mathrm{m} / \mathrm{s})$ in $\bar{x}$ and $\bar{r}$ directions, respectively

$U, V$ non-dimensional velocity components in $\bar{x}$ and $\bar{r}$ directions, respectively

\section{Greek letters}

$\alpha_{1}{ }^{*}, \alpha_{2}{ }^{*}, \beta_{1}{ }^{*}, \beta_{2}{ }^{*}, \beta_{3}{ }^{*} \quad$ rheological material moduli

$\alpha_{1}, \alpha_{2} \quad$ second-grade fluid parameters

$\beta \quad$ third-grade fluid parameter

$\alpha \quad$ thermal diffusivity $\left(\mathrm{m}^{2} / \mathrm{s}\right)$

$\beta_{T} \quad$ volumetric coefficient of thermal expansion $(1 / \mathrm{K})$

$\vartheta \quad$ kinematic viscosity $\left(\mathrm{m}^{2} / \mathrm{s}\right)$

$\mu \quad$ fluid viscosity $(\mathrm{kgm} / \mathrm{s})$

$\rho \quad$ density $\left(\mathrm{kg} / \mathrm{m}^{3}\right)$

$\tau^{*} \quad$ Cauchy stress tensor

\section{Subscripts}

$w \quad$ wall conditions

$\infty \quad$ ambient conditions

$f, g \quad$ grid levels in $(X, R)$ coordinate system

\section{Superscript}

$h \quad$ time level 


\section{INTRODUCTION}

In recent years, the free-convective heat transfer exterior to numerous geometrical bodies has become a rich area of interest in computational thermo-fluid dynamics, owing to the fact that these geometrical bodies feature widely used in process engineering systems, industrial manufacturing, geological energy extraction, nuclear waste disposal, etc. Geometrical models may include spheres, curved walls, wavy plates, cylinders, toruses, ellipses, cones etc. A comprehensive study has been done on natural convection flows which is characterized by strong thermal buoyancy force. Cylindrical geometries have gained unprecedented attention in thermal engineering and have encouraged an exceptional interest in scrutinizing the transient free-convective boundary layer flows from cylinders (pipes, tubes, ducts etc). Sparrow and Gregg [1] studied the natural convective fluid flow past an isothermal vertical cylinder. Later the non-isothermal thin vertical cylinder is done by Lee et al. [2]. Some more studies on vertical cylinder can be listed [3-4]. However, these studies did not consider the non-linear effects of stress and strain. Recently, nonNewtonian fluid theories are playing a dominant role because of its emerging applications in biomedicine technology, mining engineering, heat storage and chemical industry. This area presents a rich spectrum of nonlinear boundary value problems mainly due to the incredibly diverse range of rheological models available for simulating complex flow behavior [5-8]. Viscoelastic fluids are a particular sub-set of non-Newtonian fluids which have motivated considerable interest in the engineering and applied mathematics research communities. The categorization of these fluids is conducted by Rivlin and Ericksen [9]. Truesdell and Noll [10] have set the stress-tensor relative equations. Considering the rheological properties of these fluids, several subclasses of viscoelastic fluid are recommended. Out of differential type fluid models (i.e., the model which predicts the implicit relation between the history of stress and deformation of a gradient), the most straightforward subclass is a second-grade fluid model and is capable of predicting the normal stress differences. However, it fails to adequately explain the shear thickening and shear thinning phenomena. This failure has been overcome by third-grade fluid model, which describe the shear thickening /shear thinning phenomenon satisfactorily along with property explained by the secondgrade fluid model. Polyethylene oxides in water, manufacturing oils, slurries, molten plastics are some of the listed examples of a third-grade fluid model. The impact of inclined magnetic-field and heat transfer effects for third-grade fluid flow by considering exponentially stretching surface geometry have been revealed [11]. Further, Hayat et al. [12] explored the outcomes of third-grade 
and second-grade fluid parameters on the flow-field. Baoku et al. [13] showed the influence of thermophysical parameters and partial slip for mass and heat transfer of non-Newtonian thirdgrade fluid past a vertical plate under steady condition. Also, they found that the momentum boundary layer thickness diminishes and the thermal boundary layer thickness increases as fluid shows more shear thickening phenomenon. Anwar Bég et al. [14] presented a numerical result for dissipative free convective heat transfer of third-grade fluid from an infinite porous plate in a porous medium. Sajid et al. [15] worked on third-grade fluid using shooting method and homotopy analysis with results changing from no-slip case to full-slip case. Hayat et al. [16] studied on Soret and Dufour effects for third-grade fluid under transient condition. Ogunsola and Peter [17] investigated the impact of Arrhenius reaction and variable viscosity on third-grade fluid past a radiative surface in a porous medium. Sahoo and Poncet [18] showed the effects of a third-grade parameter and partial slip on the velocity, the temperature boundary layer, and skin-friction coefficient. Saadatmandi et al. [19] used computational numerical methods for analyzing thirdgrade fluid flow under steady condition.

Also, thermal transport in porous media has stimulated substantial interest in engineering sciences due to increasing applications in geophysics, biophysics, hydrology, computational biology, engineering (construction, petroleum and bio-remediation), drug delivery, viscous fingering in geological transport (gas and oil flows in reservoirs), chemical engineering packed beds, advanced medical imaging, gel manufacture, tribological bearings tissue replacement production and material science etc. Here, some of the research contributions on bio-medical application especially for the fluid flow through porous medium are listed such as bio-medical engineering [20], biological tissues [21], biological system and biotechnology [22], bio-heat transformation [23], biological marine modelling [24], combustion technology [25], and blood flow in biological tissues [26] etc. To know about these phenomena, it is necessary to study the behavior of flow profiles in a porous medium. A detailed study of convective flow in porous media has been done by Nield and Bejan [27], Ingham and Pop [28]. Also, many studies have been conducted including many physical phenomena to study free-convective fluid flow from a vertical cylinder through a porous medium. Minkowycz and Cheng [29] showed the possibility of having similarity solution for a linear relationship of cylinder's temperature with $x$ ( $x$ - distance along the wall of the cylinder) and also given the results for other case temperature deviations using both local non-similarity method and local similarity method. The work of Minkowycz and Cheng was 
extended by Merkin [30] and demonstrated the approximate method. At constant wall temperature, Yu“cel [31] studied free convective flow past a vertical cylinder embedded in the porous medium using a finite difference numerical method. Bassom and Rees [32] and Yih [33] both investigated free convection problem past a uniformly heated vertical cylinder in a porous medium. Chamkha et al. [34] also considered the same flow-geometry and explored the thermophoresis effects. The numerical investigation is carried out by Loganathan and Eswari [35] and found that boundary layers (velocity and thermal) increase with increased permeability parameter. For non-Darcy porous medium, free-convective heat and mass transfer analysis from an isothermal vertical cylinder was studied by Chamkha et al. [36]. Reddy studied the radiation effects over a cylinder for variable concentration and surface temperature [37].

Considering the research contributions reviewed through literature survey, an attempt has been made to study the flow analysis for the third-grade fluid past a vertical cylinder through a porous medium under unsteady conditions. Thus, it motivates to explore the third-grade fluid flow effects from a vertical cylinder within boundary layer region. The surrounding fluid temperature is taken to be lesser than the wall temperature. The numerical scheme called implicit finite difference method is used to demonstrate the transient flow effects of third-grade fluid in a porous medium.

The content of a present article is as follows: Section 2 describes the flow model with geometrical representation. Also, it gives non-dimensional formulation for third-grade fluid past an isothermal vertical cylinder in a porous medium. Next section deals with finite difference scheme and generation of a grid system. Then, the computational work along with graphical results for flow variables (both in transient and steady conditions), average skin friction and heat transfer rate can be found in section 4. It also includes comparative results between a third-grade fluid and Newtonian fluid in the porous medium. Finally, at section 5, summary and key findings of the present article are given.

\section{PROBLEM DESCRIPTION}

Unsteady flow analysis for a viscoelastic fluid of third-grade fluid model from a semi-infinite vertical cylinder of radius $r_{0}$ embedded in a porous medium under natural convection is considered. Figure 1 illustrates the flow regime with associated specific variables. A rectangular 
coordinate system, $(\bar{x}, \bar{r})$ is selected to define the geometry, where $\bar{x}$-axis is the coordinate along the axis of the cylinder (vertically increasing direction) $\& \bar{r}$-axis is the coordinate normal to vertical cylinder. " ' $T_{\infty}^{\prime}$ ' is the ambient temperature of fluid" (which is constant and equivalent to the surrounding temperature). At $t^{\prime}=0$, both the third-grade fluid and the cylinder are maintained at the same temperature i.e., $T_{\infty}^{\prime}$. Then for $t^{\prime}>0$, the cylinder surface temperature increased to $T_{w}^{\prime}\left(>T_{\infty}^{\prime}\right)$ and it is maintained after all the time. Viscous dissipation effects are not considered in the thermal equation.

\section{The constitutive equation:}

The Cauchy stress tensor $\left(\tau^{*}\right)$ constituting the third-grade fluid model with thermodynamic compatibility (given by Fosdick and Rajagopal [38]) is considered which is defined as:

$\tau^{*}=-P I+\mu S_{1}^{*}+\alpha_{1}^{*} S_{2}^{*}+\alpha_{2}^{*} S_{1}^{* 2}+\beta_{1}^{*} S_{3}^{*}+\beta_{2}^{*}\left(S_{1}^{*} S_{2}^{*}+S_{2}^{*} S_{1}^{*}\right)+\beta_{3}^{*}\left(t r S_{1}^{* 2}\right) S_{1}^{*}$

where $-P I$ is stress's spherical part (related to incompressibility constraint), $\beta_{i}^{*}(i=1,2,3)$ and $\alpha_{i}^{*}(i=1,2)$ are the material moduli (usually, these are functions depending on temperature). $S_{i}^{*}(i=1,2,3)$ are Rilvin-Ericksen tensors, mentioned as follows:

$S_{1}^{*}=(\nabla \mathrm{V})^{T^{* *}}+\nabla \mathrm{V}$

$S_{i}^{*}=\frac{d S_{i-1}^{*}}{d t}+(\nabla \mathrm{V})^{T^{* *}} S_{i-1}^{*}+S_{i-1}^{*}(\nabla \mathrm{V}), \quad i=2,3 \ldots$

where $T^{* *}$ - matrix transposition, $\mathrm{V}-$ velocity field, $\nabla$ - gradient operator and $\frac{d}{d t}$ - material derivative is formulated as $\frac{d}{d t}(\cdot)=\left(\frac{\partial}{\partial t}+\mathrm{V} \nabla\right)(\cdot)$. Also, it is assumed that all fluid motions are at Clausius-Duhem inequality and have the least value of Helmholtz free energy at equilibrium. Constraints for a third-grade fluid model are given as $\mu \geq 0 ; \quad \alpha_{1}^{*} \geq 0 ; \quad\left|\alpha_{1}^{*}+\alpha_{2}^{*}\right| \leq$ $\sqrt{24 \mu \beta_{3}^{*}} ; \quad \beta_{1}^{*}=0 ; \quad \beta_{2}^{*}=0 ; \quad \beta_{3}^{*} \geq 0$. Following substitution in Eq. (1), leads to:

$\tau^{*}=-P I+\mu S_{1}^{*}+\alpha_{1}^{*} S_{2}^{*}+\alpha_{2}^{*} S_{1}^{* 2}+\beta_{3}^{*}\left(t r S_{1}^{* 2}\right) S_{1}^{*}$ 
Under these assumptions and definitions, the natural convective boundary layer flow equations for a third-grade fluid with the implementation of Boussinesq's approximation in a Darcian porous medium are given by:

\section{Mass conservative equation:}

$\frac{\partial(\overline{r u})}{\partial \bar{x}}+\frac{\partial(\overline{r v})}{\partial \bar{r}}=0$

\section{Momentum conservative equation:}

$$
\begin{aligned}
& \rho \frac{D \bar{v}}{D t^{\prime}}=-\frac{\partial P}{\partial \bar{r}}+\frac{\partial \tau_{r \bar{x}}^{*}}{\partial \bar{x}}+\frac{\partial \tau_{r \bar{r}}^{*}}{\partial \bar{r}}+\frac{\tau_{r r}^{*}}{\bar{r}} \\
& \rho \frac{D \bar{u}}{D t}=-\frac{\partial P}{\partial \bar{x}}+\frac{\partial \tau_{\bar{x}}^{*}}{\partial \bar{x}}+\frac{\partial \tau_{\bar{x}}^{*}}{\partial \bar{r}}+\frac{\tau_{\bar{x}}^{*}}{\bar{r}}
\end{aligned}
$$

in which

$$
\begin{aligned}
\tau_{\bar{x} \bar{r}}^{*}=\tau_{\bar{r} \bar{x}}^{*}= & \mu\left(\frac{\partial \bar{u}}{\partial \bar{r}}+\frac{\partial \bar{v}}{\partial \bar{x}}\right)+\alpha_{1}^{*}\left[\frac{\partial^{2} \bar{u}}{\partial \bar{r} \partial t^{\prime}}+\frac{\partial^{2} \bar{v}}{\partial \bar{x} \partial t^{\prime}}+\bar{v} \frac{\partial^{2} \bar{u}}{\partial \bar{r}^{2}}+\bar{v} \frac{\partial^{2} \bar{v}}{\partial \bar{r} \partial \bar{x}}+\bar{u} \frac{\partial^{2} \bar{v}}{\partial \bar{x}^{2}}+\bar{u} \frac{\partial^{2} \bar{u}}{\partial \bar{x} \partial \bar{r}}+3 \frac{\partial \bar{u}}{\partial \bar{x}} \frac{\partial \bar{u}}{\partial \bar{r}}+\right. \\
& \left.\frac{\partial \bar{u}}{\partial \bar{r}} \frac{\partial \bar{v}}{\partial \bar{r}}+\frac{\partial \bar{u}}{\partial \bar{x}} \frac{\partial \bar{v}}{\partial \bar{x}}+\left(\frac{\partial \bar{w}}{\partial \bar{x}}+\frac{1}{\bar{r}} \frac{\partial \bar{u}}{\partial \bar{\varphi}}\right) \frac{\partial \bar{w}}{\partial \bar{r}}+\left(\frac{1}{\bar{r}} \frac{\partial \bar{v}}{\partial \bar{\varphi}}-\frac{\bar{w}}{\bar{r}}+\frac{\partial \bar{w}}{\partial \bar{r}}\right) \frac{\partial \bar{w}}{\partial \bar{x}}+3 \frac{\partial \bar{v}}{\partial \bar{x}} \frac{\partial \bar{v}}{\partial \bar{r}}\right]+\alpha_{2}^{*}\left[2 \frac{\partial \bar{u}}{\partial \bar{r}} \frac{\partial \bar{v}}{\partial \bar{r}}+\right. \\
& \left.2 \frac{\partial \bar{v}}{\partial \bar{r}} \frac{\partial \bar{v}}{\partial \bar{x}}+2 \frac{\partial \bar{v}}{\partial \bar{x}} \frac{\partial \bar{u}}{\partial \bar{x}}+2 \frac{\partial \bar{u}}{\partial \bar{x}} \frac{\partial \bar{u}}{\partial \bar{r}}+\left(\frac{1}{\bar{r}} \frac{\partial \bar{u}}{\partial \bar{\varphi}}+\frac{\partial \bar{w}}{\partial \bar{x}}\right)\left(\frac{\partial \bar{w}}{\partial \bar{r}}+\frac{1}{\bar{r}} \frac{\partial \bar{v}}{\partial \bar{\varphi}}-\frac{\bar{w}}{\bar{r}}\right)\right]+4 \beta_{3}^{*}\left[\left(\frac{\partial \bar{v}}{\partial \bar{r}}\right)^{2}+\right. \\
& \left.\left(\frac{\partial \bar{u}}{\partial \bar{x}}\right)^{2}+\frac{1}{2}\left(\frac{\partial \bar{u}}{\partial \bar{r}}+\frac{\partial \bar{v}}{\partial \bar{x}}\right)^{2}\right]\left(\frac{\partial \bar{u}}{\partial \bar{r}}+\frac{\partial \bar{v}}{\partial \bar{x}}\right)
\end{aligned}
$$

$$
\begin{gathered}
\tau_{\bar{r} \bar{r}}^{*}=2 \mu\left(\frac{\partial \bar{v}}{\partial \bar{r}}\right)+\alpha_{1}^{*}\left\{2 \frac{\partial^{2} \bar{v}}{\partial \bar{r} \partial t^{\prime}}+2 \bar{v} \frac{\partial^{2} \bar{v}}{\partial \bar{r}^{2}}+2 \bar{u} \frac{\partial^{2} \bar{v}}{\partial \bar{x} \partial \bar{r}}+2\left[2\left(\frac{\partial \bar{v}}{\partial \bar{r}}\right)^{2}+\frac{\partial \bar{u}}{\partial \bar{r}} \frac{\partial \bar{v}}{\partial \bar{x}}+\left(\frac{\partial \bar{u}}{\partial \bar{r}}\right)^{2}+\left(\frac{1}{\bar{r}} \frac{\partial \bar{v}}{\partial \bar{\varphi}}-\frac{\bar{w}}{\bar{r}}+\right.\right.\right. \\
\left.\left.\left.\frac{\partial \bar{w}}{\partial \bar{r}}\right) \frac{\partial \bar{w}}{\partial \bar{r}}\right]\right\}+\alpha_{2}^{*}\left\{4\left(\frac{\partial \bar{v}}{\partial \bar{r}}\right)^{2}+\left(\frac{\partial \bar{v}}{\partial \bar{x}}+\frac{\partial \bar{u}}{\partial \bar{r}}\right)^{2}+\left(\frac{1}{\bar{r}} \frac{\partial \bar{v}}{\partial \bar{\varphi}}+\frac{\partial w}{\partial \bar{r}}-\frac{\bar{w}}{\bar{r}}\right)^{2}\right\}+\beta_{3}^{*}\left\{2 \frac { \partial \overline { v } } { \partial \overline { x } } \left[4\left(\frac{\partial \bar{v}}{\partial \bar{r}}\right)^{2}+\right.\right. \\
\left.\left.2\left(\frac{\partial \bar{v}}{\partial \bar{x}}+\frac{\partial \bar{u}}{\partial \bar{r}}\right)^{2}+4\left(\frac{\partial \bar{u}}{\partial \bar{x}}\right)^{2}\right]\right\}
\end{gathered}
$$

$$
\begin{aligned}
\tau_{\bar{x} \bar{x}}^{*}=2 \mu\left(\frac{\partial \bar{u}}{\partial \bar{x}}\right)+\alpha_{1}^{*}\left\{2 \frac{\partial^{2} \bar{u}}{\partial \bar{x} \partial t^{\prime}}+2 \bar{u} \frac{\partial^{2} \bar{u}}{\partial \bar{x}^{2}}+2 \bar{v} \frac{\partial^{2} \bar{u}}{\partial \bar{x} \partial \bar{r}}+2\left[2\left(\frac{\partial \bar{u}}{\partial \bar{x}}\right)^{2}+\frac{\partial \bar{u}}{\partial \bar{r}} \frac{\partial \bar{v}}{\partial \bar{x}}+\left(\frac{\partial \bar{v}}{\partial \bar{x}}\right)^{2}+\left(\frac{1}{\bar{r}} \frac{\partial \bar{u}}{\partial \bar{\varphi}}+\right.\right.\right. \\
\left.\left.\left.\frac{\partial \bar{w}}{\partial \bar{x}}\right) \frac{\partial \bar{w}}{\partial \bar{x}}\right]\right\}+\alpha_{2}^{*}\left\{4\left(\frac{\partial \bar{u}}{\partial \bar{x}}\right)^{2}+\left(\frac{\partial \bar{v}}{\partial \bar{x}}+\frac{\partial \bar{u}}{\partial \bar{r}}\right)^{2}+\left(\frac{1}{\bar{r}} \frac{\partial \bar{u}}{\partial \bar{\varphi}}+\frac{\partial \bar{w}}{\partial \bar{x}}\right)^{2}\right\}+\beta_{3}^{*}\left\{2 \frac { \partial \overline { u } } { \partial \overline { x } } \left[4\left(\frac{\partial \bar{v}}{\partial \bar{r}}\right)^{2}+\right.\right. \\
\left.\left.4\left(\frac{\partial \bar{u}}{\partial \bar{x}}\right)^{2}+2\left(\frac{\partial \bar{u}}{\partial \bar{r}}+\frac{\partial \bar{v}}{\partial \bar{x}}\right)^{2}\right]\right\}
\end{aligned}
$$


where $(\bar{u}, \bar{v})$ is the velocity coordinate at $(\bar{x}, \bar{r}) . \tau_{\bar{r} \bar{r}}^{*}, \tau_{\bar{r} \bar{x}}^{*}, \tau_{\bar{x} \bar{r}}^{*}, \tau_{\bar{x} \bar{x}}^{*}$ are the extra stress-tensor components. The fluid-flow is assumed along vertical direction only, driven by thermal buoyancy. The conservative equations for momentum and thermal energy are given by [39-41]:

$$
\begin{aligned}
\frac{\partial \bar{u}}{\partial t^{\prime}}+\bar{u} \frac{\partial \bar{u}}{\partial \bar{x}}+\bar{v} \frac{\partial \bar{u}}{\partial \bar{r}}+\bar{u} \frac{\vartheta}{k_{1}}= & g^{\prime} \beta_{T}\left(T^{\prime}-T_{\infty}^{\prime}\right)+\vartheta \frac{\partial^{2} \bar{u}}{\partial \bar{r}^{2}}+\frac{\vartheta}{\bar{r}} \frac{\partial \bar{u}}{\partial \bar{r}}+\frac{\alpha_{1}^{*}}{\rho}\left[\frac{\partial^{3} \bar{u}}{\partial \bar{r}^{2} \partial t^{\prime}}+\frac{1}{\bar{r}} \frac{\partial^{2} \bar{u}}{\partial \bar{r} \partial t^{\prime}}+\bar{v} \frac{\partial^{3} \bar{u}}{\partial \bar{r}^{3}}\right. \\
& +2 \frac{\partial \bar{v}}{\partial \bar{r}} \frac{\partial^{2} \bar{u}}{\partial \bar{r}^{2}}+3 \frac{\partial^{2} \bar{u}}{\partial \bar{r}^{2}} \frac{\partial \bar{u}}{\partial \bar{x}}+\frac{\partial \bar{u}}{\partial \bar{r}} \frac{\partial^{2} \bar{v}}{\partial \bar{r}^{2}}+4 \frac{\partial \bar{u}}{\partial \bar{r}} \frac{\partial^{2} \bar{u}}{\partial \bar{x} \partial \bar{r}}+\frac{\bar{v}}{\bar{r}} \frac{\partial^{2} \bar{u}}{\partial \bar{r}^{2}}+\frac{\bar{u}}{\bar{r}} \frac{\partial^{2} \bar{u}}{\partial \bar{x} \partial \bar{r}}+ \\
& \left.\frac{3}{\bar{r}} \frac{\partial \bar{u}}{\partial \bar{r}} \frac{\partial \bar{u}}{\partial \bar{x}}+\bar{u} \frac{\partial^{3} \bar{u}}{\partial \bar{x} \partial \bar{r}^{2}}+\frac{1}{\bar{r}} \frac{\partial \bar{v}}{\partial \bar{r}} \frac{\partial \bar{u}}{\partial \bar{r}}\right]+\frac{\alpha_{2}^{*}}{\rho}\left[\frac{2}{\bar{r}} \frac{\partial \bar{v}}{\partial \bar{r}} \frac{\partial \bar{u}}{\partial \bar{r}}+\frac{2}{\bar{r}} \frac{\partial \bar{u}}{\partial \bar{r}} \frac{\partial \bar{u}}{\partial \bar{x}}+2 \frac{\partial^{2} \bar{u}}{\partial \bar{r}^{2}} \frac{\partial \bar{u}}{\partial \bar{x}}+\right. \\
+ & \left.2 \frac{\partial^{2} \bar{v}}{\partial \bar{r}^{2}} \frac{\partial \bar{u}}{\partial \bar{r}}+4 \frac{\partial \bar{u}}{\partial \bar{r}} \frac{\partial^{2} \bar{u}}{\partial \bar{x} \partial \bar{r}}+2 \frac{\partial \bar{v}}{\partial \bar{r}} \frac{\partial^{2} \bar{u}}{\partial \bar{r}^{2}}\right]+\frac{\beta_{3}^{*}}{\rho}\left[\frac{2}{\bar{r}}\left(\frac{\partial \bar{u}}{\partial \bar{r}}\right)^{3}+6\left(\frac{\partial \bar{u}}{\partial \bar{r}}\right)^{2} \frac{\partial^{2} \bar{u}}{\partial \bar{r}^{2}}+\right. \\
& \left.4\left(\frac{\partial \bar{u}}{\partial \bar{r}}\right)^{2} \frac{\partial^{2} \bar{u}}{\partial \bar{x}^{2}}+2 \frac{\partial \bar{u}}{\partial \bar{x}} \frac{\partial \bar{u}}{\partial \bar{r}} \frac{\partial^{2} \bar{u}}{\partial \bar{x} \partial \bar{r}}\right]
\end{aligned}
$$

The fourth term on the left-hand side of Eq. (5) represents the "Darcian drag force" in the axial direction which is a linear body force.

\section{Energy conservative equation:}

$\bar{u} \frac{\partial T^{\prime}}{\partial \bar{x}}+\bar{v} \frac{\partial T^{\prime}}{\partial \bar{r}}+\frac{\partial T^{\prime}}{\partial t^{\prime}}=\alpha \frac{\partial^{2} T^{\prime}}{\partial \bar{r}^{2}}+\frac{\alpha}{\bar{r}} \frac{\partial T^{\prime}}{\partial \bar{r}}$

The applied initial and boundary conditions:

$$
\begin{aligned}
& t^{\prime} \leq 0: \quad T^{\prime}=T_{\infty}^{\prime}, \quad \bar{v}=0, \quad \bar{u}=0 \quad \text { for all } \bar{x} \text { and } \bar{r} \\
& t^{\prime}>0: \quad T^{\prime}=T_{w}^{\prime}, \quad \bar{v}=0, \quad \bar{u}=0 \quad \text { at } \bar{r}=r_{0} \\
& T^{\prime}=T_{\infty}^{\prime}, \quad \bar{v}=0, \quad \bar{u}=0 \quad \text { at } \bar{x}=0 \\
& T^{\prime} \rightarrow T_{\infty}^{\prime}, \quad \bar{v} \rightarrow 0, \quad \bar{u} \rightarrow 0, \frac{\partial \bar{u}}{\partial \bar{r}} \rightarrow 0 \quad \text { at } \bar{r} \rightarrow \infty
\end{aligned}
$$

It is expedient to introduce the following dimensionless quantities in Eqns. (4) - (6), and also in Eqn. (7):

$$
\begin{aligned}
& X=\bar{x} / r_{0} G r ; \quad R=\bar{r} / r_{0} ; \quad U=\bar{u} r_{0} / \vartheta G r ; V=\bar{v} r_{0} / \vartheta \\
& t=\vartheta t^{\prime} / r_{0}{ }^{2} ; \quad G r={ }^{\prime} \beta_{T} r_{0}{ }^{3}\left(T_{w}^{\prime}-T_{\infty}^{\prime}\right) / \vartheta^{2} ; \quad \theta=T^{\prime}-T_{\infty}^{\prime} / T_{w}^{\prime}-T_{\infty}^{\prime} ; \quad \operatorname{Pr}=\vartheta / \alpha
\end{aligned}
$$


$D a=k_{1} / r_{0}^{2} ; \quad \alpha_{1}=\alpha_{1}^{*} / \rho r_{0}{ }^{2} ; \quad \alpha_{2}=\alpha_{2}^{*} / \rho r_{0}{ }^{2} ; \beta=\beta_{3}^{*} \vartheta / \rho r_{0}{ }^{4}$

(where $k_{1}$ is permeability of porous medium which is isotropic (i.e. same in both axial and radial directions), further all the notations can be referred at nomenclature part). Effectively the governing equations contract to the following non-dimensional form:

$\frac{\partial U}{\partial X}+\frac{\partial V}{\partial R}+\frac{V}{R}=0$

$$
\begin{aligned}
& \frac{\partial U}{\partial t}+U \frac{\partial U}{\partial X}+V \frac{\partial U}{\partial R}+U \frac{1}{D a}=\theta+\frac{\partial^{2} U}{\partial R^{2}}+\frac{1}{R} \frac{\partial U}{\partial R} \quad+\alpha_{1}\left[\frac{\partial^{3} U}{\partial R^{2} \partial t}+\frac{1}{R}\left(\frac{\partial^{2} U}{\partial R \partial t}+V \frac{\partial^{2} U}{\partial R^{2}}+U \frac{\partial^{2} U}{\partial X \partial R}+3 \frac{\partial U}{\partial X} \frac{\partial U}{\partial R}+\right.\right. \\
& \left.\left.\frac{\partial U}{\partial R} \frac{\partial V}{\partial R}\right)+V \frac{\partial^{3} U}{\partial R^{3}}+2 \frac{\partial V}{\partial R} \frac{\partial^{2} U}{\partial R^{2}}+3 \frac{\partial^{2} U}{\partial R^{2}} \frac{\partial U}{\partial X}+\frac{\partial U}{\partial R} \frac{\partial^{2} V}{\partial R^{2}}+4 \frac{\partial U}{\partial R} \frac{\partial^{2} U}{\partial X \partial R}+U \frac{\partial^{3} U}{\partial X \partial R^{2}}\right] \\
& +\alpha_{2}\left[\frac{2}{R}\left(\frac{\partial U}{\partial R} \frac{\partial V}{\partial R}+\frac{\partial U}{\partial X} \frac{\partial U}{\partial R}\right)+4 \frac{\partial U}{\partial R} \frac{\partial^{2} U}{\partial X \partial R}+2\left(\frac{\partial^{2} U}{\partial R^{2}} \frac{\partial U}{\partial X}+\frac{\partial^{2} V}{\partial R^{2}} \frac{\partial U}{\partial R}+\frac{\partial V}{\partial R} \frac{\partial^{2} U}{\partial R^{2}}\right)\right] \\
& +\beta\left[(G r)^{2}\left(\frac{2}{R}\left(\frac{\partial U}{\partial R}\right)^{3}+6\left(\frac{\partial U}{\partial R}\right)^{2} \frac{\partial^{2} U}{\partial R^{2}}\right)+2 \frac{\partial U}{\partial X} \frac{\partial U}{\partial R} \frac{\partial^{2} U}{\partial X \partial R}+4\left(\frac{\partial U}{\partial R}\right)^{2} \frac{\partial^{2} U}{\partial X^{2}}\right]
\end{aligned}
$$

$\frac{\partial \theta}{\partial t}+U \frac{\partial \theta}{\partial X}+V \frac{\partial \theta}{\partial R}=\frac{1}{P r}\left(\frac{1}{R} \frac{\partial}{\partial R}\left(R \frac{\partial \theta}{\partial R}\right)\right)$

$t=0: \quad \theta=0, \quad V=0, \quad U=0 \quad$ for all $X$ and $R$

$t>0: \theta=1, \quad V=0, \quad U=0 \quad$ at $R=1$

$$
\begin{array}{ll}
\theta=0, \quad V=0, \quad U=0 & \text { at } X=0 \\
\theta \rightarrow 0, \quad V \rightarrow 0, \quad U \rightarrow 0, \quad \frac{\partial U}{\partial R} \rightarrow 0 & \text { as } R \rightarrow \infty
\end{array}
$$

\section{FINITE DIFFERENCE SOLUTION PROCEDURE}

It is difficult to derive analytical solutions due to the strong nonlinearity of the normalized partial differential equations. A computational numerical approach is therefore chosen to solve the timedependent Eqns. (9) - (11) using the boundary conditions (12). The selected finite-difference iterative scheme which is "unconditionally stable" is known as a "Crank-Nicolson type" scheme. 
Let $J R=\frac{1}{1+(g-1) \Delta R}$. The finite difference discretized equations for Eqs. (9), (10) and (11) are as follows:

$$
\begin{aligned}
& \frac{U_{f, g}^{h+1}+U_{f, g}^{h}-U_{f-1, g}^{h+1}-U_{f-1, g}^{h}}{2 \Delta X}+\frac{V_{f, g}^{h+1}+V_{f, g}^{h}-V_{f, g-1}^{h+1}-V_{f, g-1}^{h}}{2 \Delta R}+V_{f, g}^{h+1}(J R)=0 \\
& \frac{U_{f, g}^{h+1}-U_{f, g}^{h}}{\Delta t}+\frac{U_{f, g}^{h}}{2 \Delta X}\left(U_{f, g}^{h+1}+U_{f, g}^{h}-U_{f-1, g}^{h+1}-U_{f-1, g}^{h}\right)+\frac{V_{f, g}^{h}}{4 \Delta R}\left(U_{f, g+1}^{h+1}+U_{f, g+1}^{h}-U_{f, g-1}^{h+1}-U_{f, g-1}^{h}\right) \\
& =\frac{\theta_{f, g}^{h}+\theta_{f, g}^{h+1}}{2}+\frac{J R}{4 \Delta R}\left(U_{f, g+1}^{h+1}+U_{f, g+1}^{h}-U_{f, g-1}^{h+1}-U_{f, g-1}^{h}\right) \\
& +\frac{1}{2(\Delta R)^{2}}\left(U_{f, g-1}^{h+1}+U_{f, g-1}^{h}-2 U_{f, g}^{h+1}-2 U_{f, g}^{h}+U_{f, g+1}^{h+1}+U_{f, g+1}^{h}\right) \\
& +\alpha_{1}\left[\frac{1}{4(\Delta R)^{2}(\Delta t)}\left(U_{f, g-2}^{h+1}-U_{f, g-2}^{h}-2 U_{f, g}^{h+1}+2 U_{f, g}^{h}+U_{f, g+2}^{h+1}-U_{f, g+2}^{h}\right)\right. \\
& +\frac{J R}{2(\Delta R)(\Delta t)}\left(U_{f, g+1}^{h+1}-U_{f, g+1}^{h}-U_{f, g-1}^{h+1}+U_{f, g-1}^{h}\right) \\
& +\frac{V_{f, g}^{h}}{4(\Delta R)^{3}}\left(U_{f, g+2}^{h+1}+U_{f, g+2}^{h}-2 U_{f, g+1}^{h+1}-2 U_{f, g+1}^{h}+2 U_{f, g-1}^{h+1}+2 U_{f, g-1}^{h}-U_{f, g-2}^{h+1}-U_{f, g-2}^{h}\right) \\
& +\frac{1}{2(\Delta R)^{3}}\left(V_{f, g+1}^{h}-V_{f, g-1}^{h}\right)\left(U_{f, g-1}^{h+1}+U_{f, g+1}^{h+1}-2 U_{f, g}^{h+1}-2 U_{f, g}^{h}+U_{f, g-1}^{h}+U_{f, g+1}^{h}\right) \\
& +\frac{3}{2(\Delta X)(\Delta R)^{2}}\left(U_{f, g}^{h}-U_{f-1, g}^{h}\right)\left(U_{f, g-1}^{h+1}+U_{f, g-1}^{h}-2 U_{f, g}^{h+1}-2 U_{f, g}^{h}+U_{f, g+1}^{h+1}+U_{f, g+1}^{h}\right) \\
& +\frac{1}{4(\Delta R)^{3}}\left(U_{f, g+1}^{h+1}+U_{f, g+1}^{h}-U_{f, g-1}^{h+1}-U_{f, g-1}^{h}\right)\left(V_{f, g-1}^{h}+V_{f, g+1}^{h}-2 V_{f, g}^{h}\right) \\
& +\frac{1}{2(\Delta X)(\Delta R)^{2}}\left(U_{f, g+1}^{h}-U_{f, g-1}^{h}\right)\left(U_{f, g+1}^{h+1}+U_{f, g+1}^{h}-U_{f-1, g+1}^{h+1}-U_{f-1, g+1}^{h}-U_{f, g-1}^{h+1}-\right. \\
& \left.U_{f, g-1}^{h}+U_{f-1, g-1}^{h+1}+U_{f-1, g-1}^{h}\right)+\frac{V_{f, g}^{h} J R}{2(\Delta R)^{2}}\left(U_{f, g-1}^{h+1}+U_{f, g-1}^{h}-2 U_{f, g}^{h+1}-2 U_{f, g}^{h}+U_{f, g+1}^{h+1}+\right. \\
& \left.U_{f, g+1}^{h}\right)+\frac{U_{f, g}^{h} J R}{4(\Delta R)(\Delta X)}\left(U_{f, g+1}^{h+1}+U_{f, g+1}^{h}-U_{f-1, g+1}^{h+1}-U_{f-1, g+1}^{h}-U_{f, g-1}^{h+1}-U_{f, g-1}^{h}+\right. \\
& \left.U_{f-1, g-1}^{h+1}+U_{f-1, g-1}^{h}\right)+\frac{3 J R}{4(\Delta R)(\Delta X)}\left(U_{f, g}^{h}-U_{f-1, g}^{h}\right)\left(U_{f, g+1}^{h+1}+U_{f, g+1}^{h}-U_{f, g-1}^{h+1}-U_{f, g-1}^{h}\right)
\end{aligned}
$$




$$
\begin{aligned}
& +\frac{U_{f, g}^{h}}{2(\Delta R)^{2} \Delta X}\left[U_{f, g+1}^{h+1}+U_{f, g+1}^{h}-U_{f-1, g+1}^{h+1}-U_{f-1, g+1}^{h}-2 U_{f, g}^{h+1}-2 U_{f, g}^{h}+2 U_{f-1, g}^{h+1}+2 U_{f-1, g}^{h}\right. \\
& \left.+U_{f, g-1}^{h+1}+U_{f, g-1}^{h}-U_{f-1, g-1}^{h+1}-U_{f-1, g-1}^{h}\right] \\
& \left.+\frac{J R}{8(\Delta R)^{2}}\left(V_{f, g+1}^{h}-V_{f, g-1}^{h}\right)\left(U_{f, g+1}^{h+1}+U_{f, g+1}^{h}-U_{f, g-1}^{h+1}-U_{f, g-1}^{h}\right)\right] \\
& +\alpha_{2}\left[\frac{J R}{4(\Delta R)^{2}}\left(V_{f, g+1}^{h}-V_{f, g-1}^{h}\right)\left(U_{f, g+1}^{h+1}+U_{f, g+1}^{h}-U_{f, g-1}^{h+1}-U_{f, g-1}^{h}\right)\right. \\
& +\frac{J R}{2(\Delta R)(\Delta X)}\left(U_{f, g}^{h}-U_{f-1, g}^{h}\right)\left(U_{f, g+1}^{h+1}+U_{f, g+1}^{h}-U_{f, g-1}^{h+1}-U_{f, g-1}^{h}\right) \\
& +\frac{1}{(\Delta X)(\Delta R)^{2}}\left(U_{f, g}^{h}-U_{f-1, g}^{h}\right)\left(U_{f, g-1}^{h+1}+U_{f, g-1}^{h}-2 U_{f, g}^{h+1}-2 U_{f, g}^{h}+U_{f, g+1}^{h+1}+U_{f, g+1}^{h}\right) \\
& +\frac{1}{2(\Delta R)^{3}}\left(V_{f, g-1}^{h}+V_{f, g+1}^{h}-2 V_{f, g}^{h}\right)\left(U_{f, g+1}^{h+1}+U_{f, g+1}^{h}-U_{f, g-1}^{h+1}-U_{f, g-1}^{h}\right) \\
& +\frac{1}{2(\Delta X)(\Delta R)^{2}}\left(U_{f, g+1}^{h}-U_{f, g-1}^{h}\right)\left(U_{f, g+1}^{h+1}+U_{f, g+1}^{h}-U_{f-1, g+1}^{h+1}-U_{f-1, g+1}^{h}-U_{f, g-1}^{h+1}-\right. \\
& \left.U_{f, g-1}^{h}+U_{f-1, g-1}^{h+1}+U_{f-1, g-1}^{h}\right) \\
& \left.+\frac{1}{2(\Delta R)^{3}}\left(V_{f, g+1}^{h}-V_{f, g-1}^{h}\right)\left(U_{f, g-1}^{h+1}+U_{f, g-1}^{h}-2 U_{f, g}^{h+1}-2 U_{f, g}^{h}+U_{f, g+1}^{h+1}+U_{f, g+1}^{h}\right)\right]+\beta \\
& \left\{\frac { ( G r ) ^ { 2 } ( U _ { f , g + 1 } ^ { h } - U _ { f , g - 1 } ^ { h } ) ^ { 2 } } { 4 ( \Delta R ) ^ { 3 } } \left[J R\left(U_{f, g+1}^{h}-U_{f, g-1}^{h}\right)+\frac{3}{(\Delta R)}\left(U_{f, g-1}^{h+1}-2 U_{f, g}^{h+1}+U_{f, g+1}^{h+1}+U_{f, g-1}^{h}-\right.\right.\right. \\
& \left.\left.\left.2 U_{f, g}^{h}+U_{f, g+1}^{h}\right)\right]\right\}+\left\{\frac { ( U _ { f , g + 1 } ^ { h } - U _ { f , g - 1 } ^ { h } ) } { ( \Delta X ) ^ { 2 } ( \Delta R ) ^ { 2 } } \left[\left(U_{f-1, g}^{h}-2 U_{f, g}^{h}+U_{f+1, g}^{h}\right)\left(U_{f, g+1}^{h}-U_{f, g-1}^{h}\right)+\right.\right. \\
& \frac{1}{8}\left(U_{f, g}^{h}-U_{f-1, g}^{h}\right)\left(U_{f, g+1}^{h+1}-U_{f-1, g+1}^{h+1}-U_{f, g-1}^{h+1}+U_{f-1, g-1}^{h+1}+U_{f, g+1}^{h}-U_{f-1, g+1}^{h}-\right. \\
& \left.\left.\left.U_{f, g-1}^{h}+U_{f-1, g-1}^{h}\right)\right]\right\}-\frac{1}{\mathrm{Da}} \frac{U_{f, g}^{h+1}+U_{f, g}^{h}}{2}
\end{aligned}
$$

$$
\frac{\theta_{f, g}^{h+1}-\theta_{f, g}^{h}}{\Delta t}+\frac{U_{f, g}^{h}}{2 \Delta X}\left(\theta_{f, g}^{h+1}-\theta_{f-1, g}^{h+1}+\theta_{f, g}^{h}-\theta_{f-1, g}^{h}\right)+\frac{V_{f, g}^{h}}{4 \Delta R}\left(\theta_{f, g+1}^{h+1}-\theta_{f, g-1}^{h+1}+\theta_{f, g+1}^{h}-\theta_{f, g-1}^{h}\right)
$$




$$
\begin{aligned}
= & \frac{1}{2 \operatorname{Pr}(\Delta R)^{2}}\left(\theta_{f, g-1}^{h+1}-2 \theta_{f, g}^{h+1}+\theta_{f, g+1}^{h+1}+\theta_{f, g-1}^{h}-2 \theta_{f, g}^{h}+\theta_{f, g+1}^{h}\right) \\
& +\frac{J R}{4 \operatorname{Pr} \Delta R}\left(\theta_{f, g+1}^{h+1}-\theta_{f, g-1}^{h+1}+\theta_{f, g+1}^{h}-\theta_{f, g-1}^{h}\right)
\end{aligned}
$$

A rectangular grid system with $X_{\min }=0, X_{\max }=1, R_{\min }=1$ and $R_{\max }=20$ (where $R_{\max }$ implies to $R=\infty$ ) is selected.

The finite difference technique commences with the computation of the thermal conservative Eq. (11), resulting the temperature field. Then, the procedure follows to solve the momentum transport and mass conservative equations (10) and (9), yielding the velocity field. At the $(h+1)^{\text {th }}$ stage Eqns. (15) and (14) are stated below in tridiagonal and penta-diagonal forms:

$$
\begin{gathered}
A_{1} \omega_{f, g-1}^{h+1}+B_{1} \omega_{f, g}^{h+1}+C_{1} \omega_{f, g+1}^{h+1}=D_{1} \\
A_{2} \varphi_{f, g-2}^{h+1}+B_{2} \varphi_{f, g-1}^{h+1}+C_{2} \varphi_{f, g}^{h+1}+D_{2} \varphi_{f, g+1}^{h+1}+E_{2} \varphi_{f, g+2}^{h+1}=F_{2}
\end{gathered}
$$

where $\omega$ and $\varphi$ indicate $\theta$ and $U$, respectively. Thus, Eqns. (14) - (15) at each interior mesh point on a specific $f$-level involve a system of penta-diagonal and tridiagonal equations. A comprehensive study of the finite difference method can be found in the literature [42-43].

\section{Validation of the numerical scheme:}

A consistent and economic grid system is selected using grid-independent test for different sizes

of a grid. The values of the Nusselt number $(\overline{N u})$ and average skin-friction coefficient $\left(\bar{C}_{f}\right)$ on the boundary $R=1$ are presented in Table 1 . It is observed from Table 1 that grid size of $100 \mathrm{X} 500$ gives an adequate accuracy to study the present problem. Likewise, a time-independence test has been conducted for different sizes of a time step, as shown in Table 2. The effective particular time step size $\Delta t(t=h \Delta t, h=0,1,2, \ldots)$ is fixed as 0.01 . Further, the scheme is "unconditionally stable where the local truncation error is $O\left(\Delta t^{2}+\Delta Y^{2}+\Delta X\right)$ and it tends to zero as $\Delta t, \Delta Y$ and $\Delta X$ tends to zero". 


\section{RESULTS AND DISCUSSION}

The transient behavior of $U$ and $\theta$ are studied, and their values are explained at different locations. The steady-state $U$ and $\theta$ profiles are discussed along the direction of $R$. The results for the case of Newtonian fluids in the absence of a porous medium $\left(\alpha_{1}=\alpha_{2}=\beta=0\right)$ are in comparison with those of Lee et al. [2] (refer Fig. 2a). Also, one more comparative result for Newtonian fluid flow (i.e., $\alpha_{1}=\alpha_{2}=\beta=0$ ) is conducted by neglecting the effect of the third grade viscoelastic parameter in the current model and comparing with the results of Rani and $\operatorname{Kim}$ [44] for $\gamma=\lambda=0$ (refer Fig. 2b). Generally, a close correlation is achieved which endorses the accuracy of the present numerical code. The simulated results are represented to describe the variation of the dimensionless flow variables along with heat transport coefficients and average skin-friction for different thermophysical control parameters and are discussed in the following subsections.

The range of third-grade fluid parameters $\alpha_{1}, \alpha_{2}, \alpha_{3}$ is taken from the inequality given by Clasusius-Duhelm with least value of Helmholtz free energy at equilibrium and the conditions of Eq. (8) are as follows. The stability and thermodynamic criteria of third-grade fluid is considered and it is determined that boundedness and stability nature of third-grade fluid can be deliberated as case of second-grade fluid (scrutinized by Dunn and Fosdick [45], for $\beta_{3}^{*}=0$ gives the constitutive equation for a second-grade Reiner-Rivlin fluid). Also, Fosdick and Straughan [46] revealed that the restriction $\alpha_{1}{ }^{*}<0$ provides non-physical results (i.e., "quite arbitrary flows with instability and unboundedness") and asymptotic stability for $\alpha_{1}^{*} \geq 0$.

The considered value of $G r$ (Grashof number) is 10. For higher $G r$ value the viscous force is comparably negligible from the 'thermal buoyancy' forces. When buoyancy forces massively 
exceed the viscous forces, fluid flow will eventually morph to a turbulent one with transitional disturbances growing quickly. However, the lower values of $G r$ encourages the flow that moves upward direction causing smooth turns around the heated vertical cylinder. Buoyancy therefore when judiciously controlled can be used to strategically modify flow patterns which is beneficial to thermal coating applications and achieves more homogenous coating thicknesses. The Prandtl number is fixed as $\operatorname{Pr}=0.63$ (particularly represents the oxygen). Also, the maximum value of Darcy number is 5 which indicates the greater effect of permeability of the medium and hence significant reduction in the bulk matrix drag of the porous medium fibers.

\subsection{Flow Variables}

\section{Velocity:}

The simulated transient non-dimensional velocity $(U)$ versus time $(t)$ at different locations $(1,1.83)$ and $(1,5.82)$ by varying the third-grade fluid parameter $(\beta)$ and the Darcy number (Da) are depicted in Fig. 3 and Fig. 4, respectively. The $U$ profiles, in Figs. 3 and 4 are chosen in the neighbourhood and far distance from the heated cylindrical surface, respectively. Fig. 3a displays the transient velocity profile for variation in $\beta$ value with $\mathrm{Da}=5, \alpha_{1}=\alpha_{2}=0.2$. Similarly, Fig. $3 b$ shows the graphical results for variation in Da value with $\beta=0.1, \alpha_{1}=\alpha_{2}=0.2$. At all localities, it is noticed that velocity curve is enhanced with time, achieves temporal maxima and then lastly reaches the time-independent state. For fixed values of $\alpha_{1}=\alpha_{2}=0.2, \beta=0.5$ and Da $=5$, it is evident that the velocity increases monotonically with time, attains temporal maxima and at last reaches the asymptotic steady-state (refer Fig. 3a). Also, when $t<<1$, it can be observed that the heat transfer conduction takes dominating rule over the convection. Consequently, there befalls a time phase where the heat transfer rate is swayed by the increase of natural convection hence accelerating the flow of third-grade fluid with respect to time. For all values of Da with fixed $\beta=0.1$, Fig. $3 b$ shows that it has the similar unsteady behaviour as the observations made in Fig. 3a pertaining to $\beta$. It is noticed in Fig. 3a that initially $(t<1)$ unsteady $U$ profile coincides with each other and then deviates for a later time $(t \geq 1)$. Also, the magnitude of the velocity attained increases as Da is enhanced. Since, the permeability of the porous medium (i.e., measure 
of ease with which the fluid can move inside the porous medium) and the Darcy number (Da), both are in direct relation i.e., bigger Darcy number implies to greater permeability of the porous medium and an associated depletion in bulk matrix drag of the porous medium fibers. Hence, as Darcy number increases, the flow gets accelerated. Also, from Fig. 3a, with increasing $\beta$ value time to attain the temporal maxima decreases, but for increasing $\mathrm{Da}$, time to needed for attaining the temporal maxima increases shown in Fig. 3b. For augmenting value of the $\beta$ with fixed $\mathrm{Da}=$ $5, \alpha_{1}=\alpha_{2}=0.2$, the magnitude of velocity is decreasing close to hot surface (refer Fig. 3a) and it is increasing at a distance far away from the hot surface (refer Fig. 4a). The decrement in the velocity of the fluid at the hot wall of the cylinder is due to increasing $\beta$ i.e., it pronounces the viscoelastic effect (i.e. the relative influential action of elastic and viscous forces). It dominates the effect of viscosity and recedes the fluid elastic nature hence a deceleration in the fluid velocity is observed (Fig. 3a). Whereas the variation of Da value has similar effects at all the locations (refer Figs. 3b and 4b).

Also, from Fig. 5, these $U$ profiles relating to $\beta$ and Da are examined next. The steady-state non-dimensional velocity $(U)$ versus non-dimensional radial coordinate $(R)$ is plotted in Fig. 5 for various values of $\beta$ and Da. Under steady conditions with fixed $\alpha_{1}=\alpha_{2}=0.2$, Fig. 5a displays the velocity profile for variation of $\beta$ value with $\mathrm{Da}=5$ and Fig. $5 \mathrm{~b}$ shows velocity variations for the influence of Da number with $\beta=0.1$. Clearly, the velocity profiles begin with zero value adjacent to cylindrical wall, attain the maximum and subsequently fall to zero in the $R$ direction. It is also observed that close to cylindrical surface, the magnitude of $U$ rises quickly as the $R$ value increase from $R_{\min }$ along the axis of the cylinder. From the Fig. 5a, it is perceived that for increasing $\beta$, the velocity decreases in a neighbourhood of the cylindrical wall and the trend is reversed after for some $R$, i.e., at distance far away from the wall. It is witnessed from Fig. $5 \mathrm{~b}$ that, near to the hot wall, the velocity increases as Da increases. Since the increased Da value promotes the growth of the permeability of the medium, this manifests in a depletion in the bulk impedance of the solid fibers (it is observed that the pure fluid state can be obtained by taking infinite permeability, i.e., vanishing Darcian drag force in the momentum equation). 


\section{Temperature:}

Figure 6 shows the transient non-dimensional temperature $(\theta)$ against time $(t)$ at $(1,1.15)$. The influence of variation of $\beta$ with fixed $\mathrm{Da}=5, \alpha_{1}=\alpha_{2}=0.2$ is shown in Fig. 6a. Similarly effect of Da with fixed $\beta=0.1, \alpha_{1}=\alpha_{2}=0.2$ is shown in Fig. $6 \mathrm{~b}$. At initial period, these profiles show increasing nature against time and then reach time-independent state. At all the positions, this unsteady behavior of temperature is observed. From Fig. 6a, it is noticed that the temporal maximum increase as the third-grade fluid parameter increases indicating that greater viscoelastic effect aids to heat up the regime due to enhanced particle collision and to increase thermal boundary layer thickness. Converse response for thermal energy distributions in transient condition can be found in Fig. $6 \mathrm{~b}$ for all values of Da with $\beta=0.1, \alpha_{1}=\alpha_{2}=0.2$, i.e., as Da increases the temperature decreases. The thermal conduction diminishes for a higher value of permeability (i.e., increased Da value) of porous medium as a result of decreased temperature. Hence it cools the regime and results in shrinkage of a thermal boundary layer.

The steady-state temperature $(\theta)$ against the $R$ for different values of $\beta$ and Da are presented in Fig. 7. From Fig. 7a and $7 \mathrm{~b}, \theta$ profiles start with a temperature of the hot surface and then continuously decay to zero value along $R$. Figure 7a displays the influence of third-grade fluid parameter $(\beta)$ with $\mathrm{Da}=5, \alpha_{1}=\alpha_{2}=0.2$. It also shows the viscoelastic effect of third-grade fluid, i.e., as $\beta$ increases temperature profile increases. Greater viscoelasticity (higher $\beta$ ) values slow down the fluid motion and this encourages thermal diffusion. Also, greater $\beta$ values cause to increase the steady-state time. From Fig.7b, it is revealed that the steady-state time decreases as $D a$ increases.

\subsection{Friction and Heat Transport Coefficients}

For known flow-field profiles under steady and unsteady conditions, transport coefficients for the third-grade fluid are essential parameters to study free-convective heat transfer problems. The nondimensional average skin friction and heat transport coefficients are given by

$\overline{C_{f}}=\int_{0}^{1}\left(\frac{\partial U}{\partial R}\right)_{R=1} d X$ and $\overline{N u}=-\int_{0}^{1}\left(\frac{\partial \theta}{\partial R}\right)_{R=1} d X$ respectively. 
Figure 8 reveals the results for $\overline{C_{f}}$ for various values of $\beta$ and Da. At an initial phase of time, these figures indicate that skin friction $\left(\overline{C_{f}}\right)$ increases monotonically against the time $(t)$ finally achieving the time-independent state. Also, it is evident that velocity has lesser value as shown in Fig. 3, which is reflected in Fig. 8 for average skin friction coefficient. It is seen that for increasing value of $\beta$, the $\overline{C_{f}}$ decreases shown in Fig. $8 \mathrm{a}$ and reverse tendency can be observed for escalating Da which is shown in Fig. 8b. The observations made in Fig. 8a are obvious since augmenting $\beta$ (third-grade parameter) value increases the fluid viscosity hence hinders the fluid flow causing a decrease in velocity within the boundary layer. Increased Da (i.e., higher value of permeability) value causes to decrease the drag force of porous medium and hence increasing velocity of fluid intern increasing the $\overline{C_{f}}$ value.

The graphical results for $\overline{N u}$ against time for different values of $\beta$ and Da are revealed in Fig. 9. At early time phase for all $\beta$ and Da values, the $\overline{N u}$ curve decreases drastically, later reaches the steady-state. The $\overline{N u}$ values augment with decreasing $\beta$ or increasing Da and results are reflected graphically in Fig. 9a and Fig. 9b, respectively. The results from Fig. 9a are true since rising $\beta$ values cause to increase the flow-field temperature near to the hot cylindrical surface and decelerating the fluid flow, hence there is negatively increase in heat transfer rate to the wall, i.e., $\overline{N u}$. The temperature gradient effects the variation in $\overline{N u}$. From Fig. 9a, $\overline{N u}$ is hardly affected by variation of $\beta$ value, i.e., decrease in the fluid viscosity does not noticeably modify heat transfer rates. Similarly, in Fig. 9b, the effect of permeability of porous medium on heat transfer by increasing $D a$ value is reflected. Since greater the permeability effect of porous medium cools the flow regime, this in turn enhances the heat transfer near the hot surface.

\section{Comparison results}

The contours $(U$ and $\theta$ ) for Newtonian and third-grade fluid flows for constant values of $\operatorname{Pr}=0.63$, and $\mathrm{Da}=5$ in a two-dimensional porous medium are presented in Fig. 10. At any locations excluding the points at boundary $(R=1, R=20 \& X=0)$, the velocity flow profile for third-grade fluid is observed to be smaller than that of a Newtonian fluid. However, a reverse trend is observed for temperature profiles. For steady-state state velocity and temperature contours, the thickness of 
thermal and hydrodynamic boundary layers for non-Newtonian third-grade fluid varies slightly compared to Newtonian fluid flow.

\section{CONCLUDING REMARKS}

Transient flow analysis has been conducted for the flow of time-dependent incompressible freeconvective third-grade fluid from isothermal vertical cylinder embedded in the porous medium. The normalized conservative equations for thermal and momentum conservation are solved by performing Crank-Nicolson scheme. The simulations are run by varying non-dimensional quantities such as third-grade parameter and Darcy number to show transient effects of third- grade viscoelastic fluid dynamics in a porous medium.

$>$ In the transient graphs, the velocity exhibits an inverse relationship with third-grade viscoelastic parameter and a direct relationship with Darcy parameter. The reverse trends are computed for temperature, i.e., the temperature increases with increasing third-grade viscoelastic and decreasing Darcy number; however, the modifications are significantly less dramatic than for the velocity distribution.

$>$ In the steady-state graphs, the time which elapses to attain the time-independent state increases with elevation in values of the third-grade viscoelastic parameter whereas it is reduced with an increase in Darcy number.

$>$ The average skin friction (momentum transport) coefficient is augmented with larger value of Darcy number whereas it is decreased with greater value of third-grade viscoelastic parameter. Similarly, the averaged heat transport coefficient is decreased with increasing values of third-grade viscoelastic parameter and it is increased with higher values of the Darcy number.

$>$ The Crank-Nicolson scheme is an excellent numerical methodology for simulating unsteady non-Newtonian external convection flows and holds significant promise for future simulations including alternate viscoelastic models, electro-conductive polymer flows, viscoplastic enrobing fluids, micro-structural rheological coating liquids etc. Efforts in this direction are currently underway and will be communicated imminently. 


\section{ACKNOWLEDGEMENTS}

The authors are extremely grateful to the reviewers for their insightful comments which have aided in improving the clarity of the work.

\section{REFERENCES}

1. Sparrow, E. M. and Gregg, J. L. "Laminar free convection heat transfer from the outer surface of a vertical circular cylinder", ASME Journal of Heat Transfer 78(8), pp. 1823-1829 (1956).

2. Lee, H. R., Chen, T. S. and Armaly, B. F. "Natural convection along slender vertical cylinders with variable surface temperature", ASME Journal of Heat Transfer 110, pp. 103108(1988).

3. M. M. Gnaneswara Reddy and N. Bhaskar Reddy, "Thermal radiation and mass transfer effects on MHD free convection flow past a vertical cylinder with variable surface temperature and concentration", Journal of Naval Architecture and Marine Engineering, 6, pp. 1-15, 2009.

4. Gnaneswara Reddy Machireddy, "Chemically reactive species and radiation effects on MHD convective flow past a moving vertical cylinder", Ain Shams Engineering Journal (2013) 4, 879-888.

5. M. Gnaneswara Reddy, O.D. Makinde, "Magnetohydrodynamic peristaltic transport of Jeffrey nanofluid in an asymmetric channel”, Journal of Molecular Liquids 223 (2016) $1242-1248$.

6. Machireddy Gnaneswara Reddy, J. Manjula and P. Padma, "Mass transfer flow of MHD radiative tangent hyperbolic fluid over a cylinder: a numerical study", Int. J. Appl. Comput. Math (2017) Volume 3 (1), pp. 447-4723.

7. O. D. Makinde, M. Gnaneswara Reddy and K. Venugopal Reddy, "Effects of thermal radiation on MHD peristaltic motion of Walters-B fluid with heat source and slip conditions", Journal of Applied Fluid Mechanics, Vol. 10, No. 4, pp. 1105-1112, 2017. 
8. Reddy, M. Gnaneswara, Kumari, P. Vijaya, Padma, P. "Effect of thermal radiation on MHD casson nano fluid over a cylinder", Journal of Nanofluids, Volume 7, Number 3, June 2018, pp. 428-438(11).

9. Rivlin, R.S. and Ericksen, J.L. "Stress deformation relations for isotropic materials", J. Rational Mech. Anal, 4, pp. 323 (1955).

10. Truesdell, C. and Noll, W. "The non-linear field theories of mechanics", in: Handbuch. Phys, Vol. III/3, Springer, New York, (1965).

11. Hayat, T., Shafiq, A., Alsaedi, A. and Asghar, S. "Effect of inclined magnetic field in flow of third grade fluid with variable thermal conductivity", AIP Advances, 5, pp. 08710815(2015).

12. Hayat, T., Shafiq, A. and Alsaedi, A. "MHD axisymmetric flow of third-grade fluid by a stretching cylinder", Alexandria Engineering Journal, 54, pp. 205 - 212 (2015).

13. Baoku, I.G., Olajuwon, B.I. and Mustapha, A.O. "Heat and mass transfer on a MHD third grade fluid with partial slip flow past an infinite vertical insulated porous plate in a porous medium", International Journal of Heat and Fluid Flow, 40, pp. 81-88(2013).

14. Anwar Bég, O., Takhar, H.S., Bhargava, R., Rawat, S. and Prasad, V.R. "Numerical study of heat transfer of a third-grade viscoelastic fluid in non-Darcy porous media with thermophysical effects”, IOP Publishing, Physica Scripta, 77, pp. 065402-11 (2008).

15. Sajid, M., Ahmad, M., Ahmad, I., Taj, M. and Abbasi, A. “Axisymmetric stagnation-point flow of a third-grade fluid over a lubricated surface", Advances in Mechanical Engineering, 7, pp. 1-8 (2015).

16. Hayat, T., Awais, M., Asghar, S. and Obaidat, S. "Unsteady flow of third grade fluid with Soret and Dufour effects", ASME Journal of Heat Transfer, 134, pp. 062001 - 7 (2012).

17. Ogunsola, A.W. and Peter, B.A. "Effect of Variable Viscosity on Third Grade Fluid Flow over a Radiative Surface with Arrhenius Reaction", International Journal of Pure and Applied Sciences and Technology, 22, pp. 1-8(2014).

18. Sahoo, B. and Poncet, S. "Flow and heat transfer of a third-grade fluid past an exponentially stretching sheet with partial slip boundary condition", International Journal of Heat and Mass Transfer, 54, pp. 5010-5019(2011). 
19. Saadatmandi, A., Sanatkar, Z. and Toufighi, S. P. "Computational methods for solving the steady flow of a third-grade fluid in a porous half space", Applied Mathematics and Computation, 298, pp. 133-140(2017).

20. Khanafer, K. and Vafai, K. "The role of porous media in biomedical engineering as related to magnetic resonance imaging and drug delivery", Heat Mass Transfer, 42, pp. 939953(2006).

21. Khanafer, K., AlAmiri, A., Pop, I. and Bull, J.L. "Flow and Heat Transfer in Biological Tissues: Application of Porous Media Theory", Emerging Topics in Heat and Mass Transfer in Porous Media, 22, pp. 237-259(2008).

22. Vafai, K. "Porous Media Applications in Biological Systems and Biotechnology", CRC Press. Taylor \& Francis Croup. New York, (2010).

23. Khaled, A.R.A. and Vafai, K. "The role of porous media in modeling flow and heat transfer in biological tissues", International Journal of Heat and Mass Transfer, 46, pp. 49895003(2003).

24. Khalili1, A., Liu, B., Javadi, K., Morad, M.R., Matyka, M., Kindler, K., Stocker, R., and Koza, Z. "Application of porous media theories in marine biological modeling”, In book: Porous Media, pp. 365-398(2010).

25. Mujeebu, M.A., Abdullah, M.Z., Abu Bakar, M.Z., Mohamad, A.A. and Abdullah, M.K. "Applications of porous media combustion technology - A review", Applied Energy, 86, pp. 1365-1375(2009).

26. Nakayama, A. and Kuwahara, F. "A general bioheat transfer model based on the theory of porous media", International Journal of Heat and Mass Transfer, 51, pp. 31903199(2008).

27. Nield, D. A. and Bejan, A. "Convection in Porous Media", Springer, New York, (2013).

28. Ingham, D. B. and Pop, I. "Transport Phenomena in Porous Media", Elsevier Science, Oxford, (1998).

29. Minkowycz, W.J. and Cheng, P. "Free convection about a vertical cylinder embedded in a porous medium”, Int. J. Heat Mass Transfer, 19, pp. 805-13(1976).

30. Merkin, J.H. "Free convection from a Vertical Cylinder Embedded in a Saturated Porous Medium”, Acta Mechanica, 62, pp. 19-28(1986). 
31. Yu "cel, A. "The influence of injection or withdrawal of fluid on free convection about vertical cylinder in a porous medium”, Numer. Heat Transfer, 20, pp. 483-93(1984).

32. Bassom, A.P. and Rees, D.A.S. "Free convection from a heated vertical cylinder in a fluid-saturated porous medium", Acta Mechanica, 116, pp. 139(1996).

33. Yih, K.A. "Radiation effect on natural convection over a vertical cylinder embedded in porous media", Int. Comm. Heat Mass Transfer, 26, pp. 259-67(1999).

34. Chamkha, A.J., Jaradat, M. and Pop, I. "Thermophoresis free convection from a vertical cylinder embedded in a porous medium", Int. J. Appl. Mech. Eng., 9, pp. 471-81(2004).

35. Loganathan, P. and Eswari, B. "Natural Convective Flow over moving Vertical Cylinder with Temperature Oscillations in the Presence of Porous Medium", Global Journal of Pure and Applied Mathematics, 13, pp. 839-855 (2017).

36. Chamkha, A.J, EL-Kabeir, S.M.M. and Rashad, A.M. "Heat and mass transfer by nonDarcy free convection embedded in porous media with a temperature-dependent viscosity", International Journal of Numerical Methods for Heat \& Fluid Flow, 21, pp. 847863(2011).

37. Machireddy Gnaneswara Reddy, "Radiation effects on MHD natural convection flow along a vertical cylinder embedded in a porous medium with variable surface temperature and concentration", Frontiers in Heat and Mass Transfer (FHMT), 5, 4 (2014).

38. Fosdick, R. L. and Rajagopal, K. R. "Thermodynamics and stability of fluids of third grade", Proceedings of the Royal Society of London. Series A, 369, pp. 351 - 377 (1980).

39. Hayat, T., Nazar, H., Imtiaz, M., Alsaedi, A. and Ayub, M. “Axisymmetric squeezing flow of third grade fluid in presence of convective conditions", Chinese Journal of Physics, 55, pp. 738-754 (2017).

40. Hayat, T., Mustafa, M. and Asghar, S. "Unsteady flow with heat and mass transfer of a third-grade fluid over a stretching surface in the presence of chemical reaction", Nonlinear Analysis: Real World Applications, 11, pp. 3186-3197 (2010).

41. Sajid, M., Hayat, T. and Asghar, S. "Non-similar solution for the axisymmetric flow of a third-grade fluid over a radially stretching sheet”, Acta Mechanica, 189, pp. 193-205 (2007). 
42. Rani, H. P., Reddy, G. J. and Kim, C. N. "Transient analysis of diffusive chemical reactive species for couple stress fluid flow over vertical cylinder", Applied Mathematics and Mechanics, 34, pp. 985 - 1000 (2013).

43. Reddy, G. J., Hiremath, A. and Kumar, M. "Computational modeling of unsteady thirdgrade fluid flow over a vertical cylinder: a study of heat transfer visualization”, Results in Physics 8 pp. 671-682 (2018).

44. H. P. Rani and C. N. Kim, Transient convection on a vertical cylinder with vaiable viscosity and thermal conductivity, AIAA J. Thermophysics Heat Transfer, 22, 254-261 (2008).

45. Dunn, J. E. and Rajagopal, K. R. "Fluids of differential type: critical review and thermodynamic analysis", International Journal of Engineering Science, 33, pp. 689 - 729 (1995).

46. R. L. Fosdick and B. Straughan, Catastrophic instabilities and related results in a fluid of third grade. Int. J. Non-Linear Mech. 16, 191(1981). 
Table1. Grid independent test:

\begin{tabular}{|l|c|c|}
\hline Grid size & $\bar{C}_{f}$ values & $\overline{N u}$ values \\
& for $\operatorname{Pr}=0.63, \beta=0.1$ and $\mathrm{Da}=5.0$. & for $\operatorname{Pr}=0.63, \beta=0.1$ and $\mathrm{Da}=5.0$. \\
\hline $25 \times 125$ & 0.0918315 & 0.4612852 \\
\hline $50 \times 250$ & 0.0905507 & 0.4524799 \\
\hline $100 \times 500$ & 0.0911236 & 0.4481122 \\
\hline $200 \times 1000$ & 0.0883496 & 0.4454812 \\
\hline
\end{tabular}

Table2. Time independent test:

\begin{tabular}{|c|c|c|}
\hline $\begin{array}{l}\text { Time step } \\
\text { size }(\Delta t)\end{array}$ & $\begin{array}{c}\bar{C}_{f} \text { values } \\
\text { for } P r=0.63, \beta=0.1 \text { and } \mathrm{Da}=5.0 .\end{array}$ & $\begin{array}{c}\overline{N u} \text { values } \\
0.1\end{array}$ \\
\hline & 0.15866445 & 0.6614244 \\
\hline 0.08 & 0.15302148 & 0.6434421 \\
\hline 0.05 & 0.13995002 & 0.6004548 \\
\hline 0.02 & 0.11203902 & 0.5090560 \\
\hline & & \\
\hline & & \\
\hline
\end{tabular}




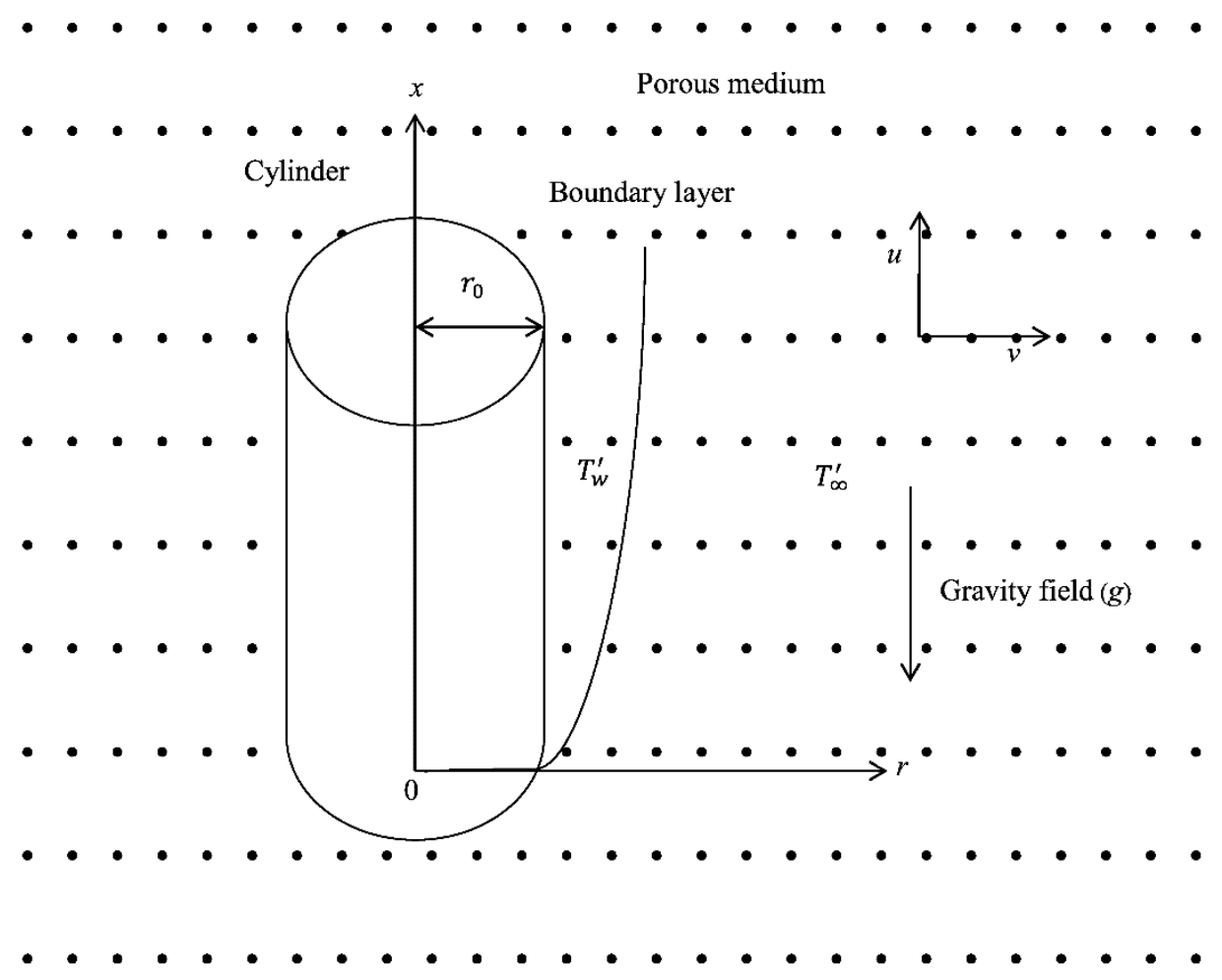

Fig. 1. Graphical representation of present problem. 


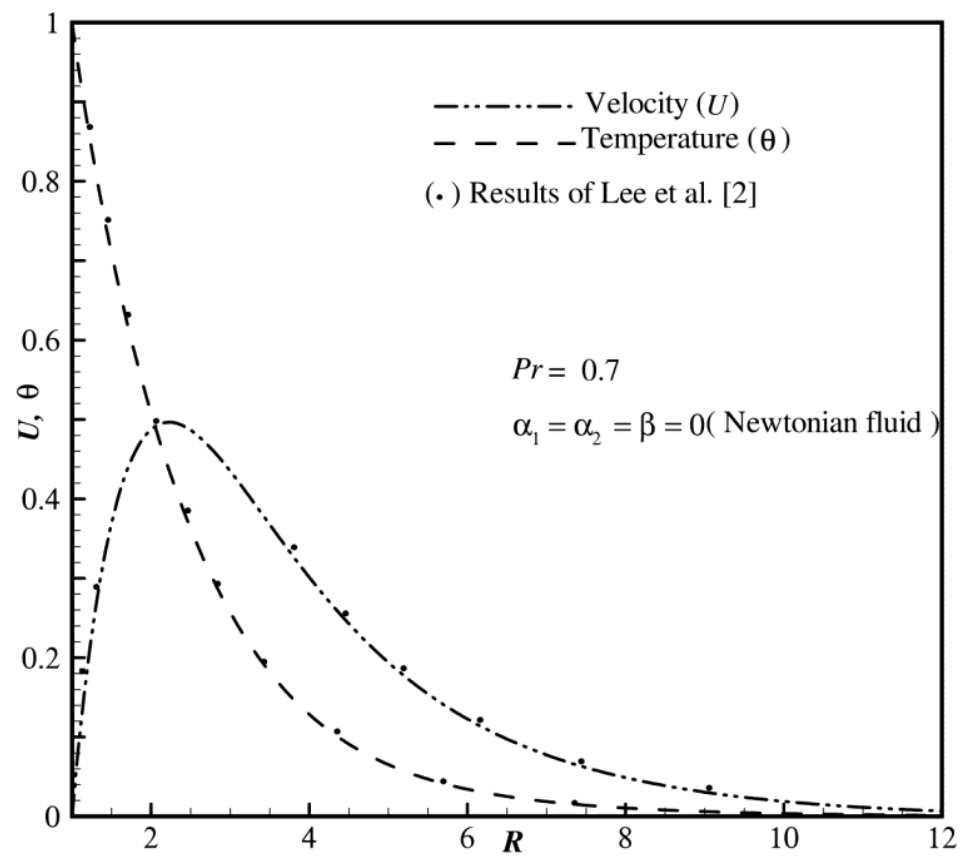

(a)

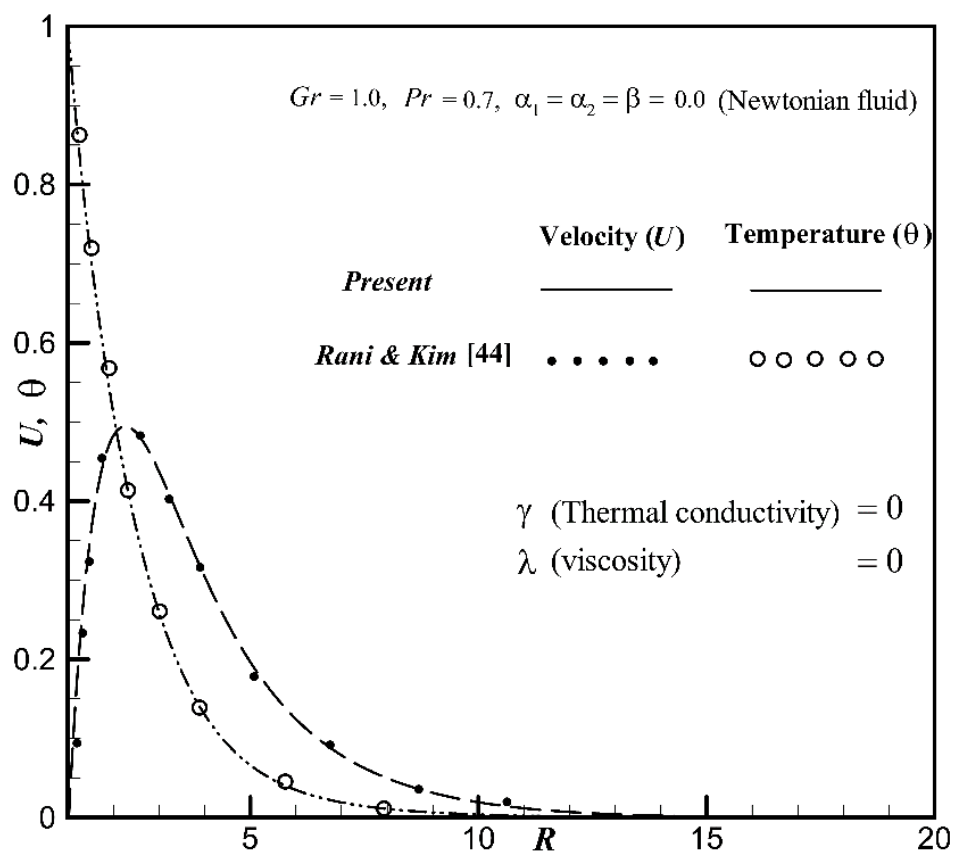

(b)

Fig. 2. Comparison of the velocity and temperature profiles. 


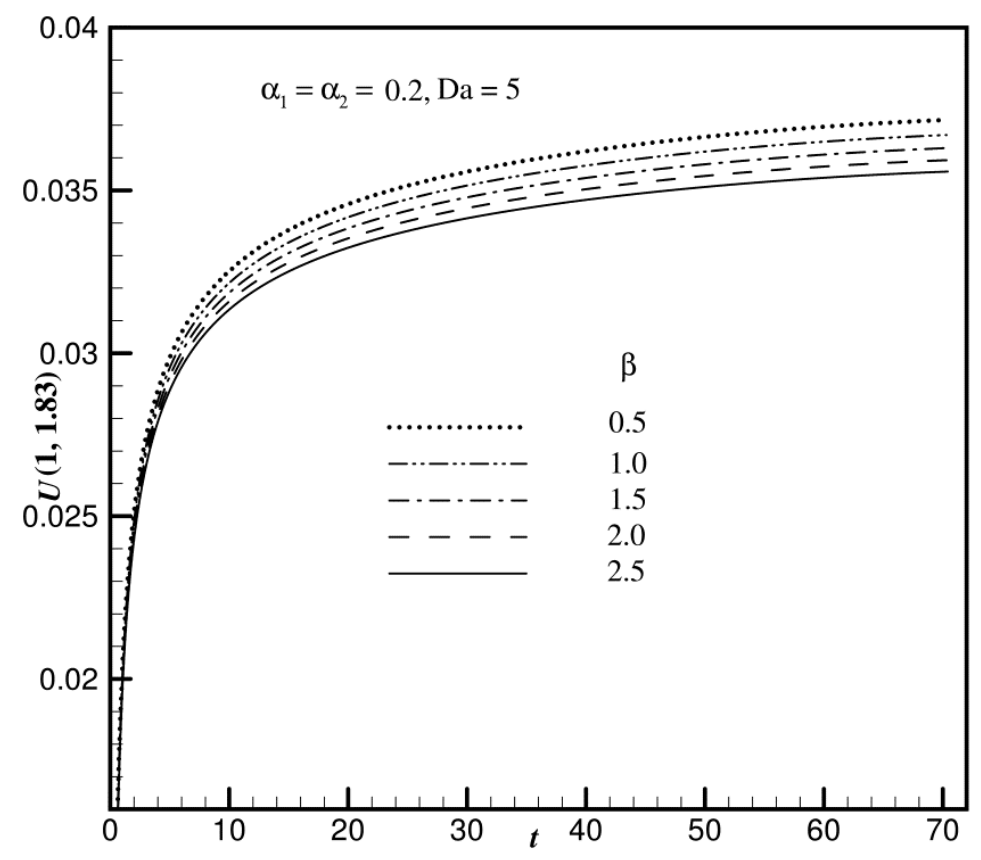

(3a)

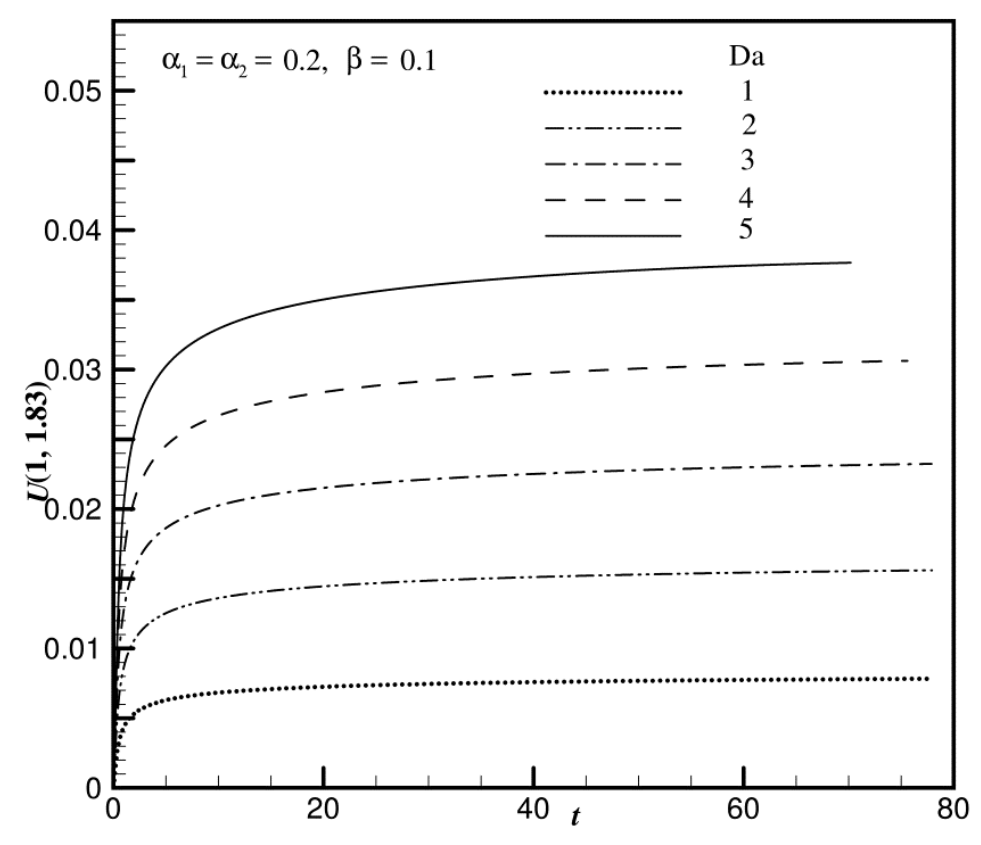

(3b)

Fig. 3. Time-dependent velocity profile $(U)$ versus time $(t)$ at the point $(1,1.83)$ for the effect of (a) $\beta ; \&$ (b) Da. 


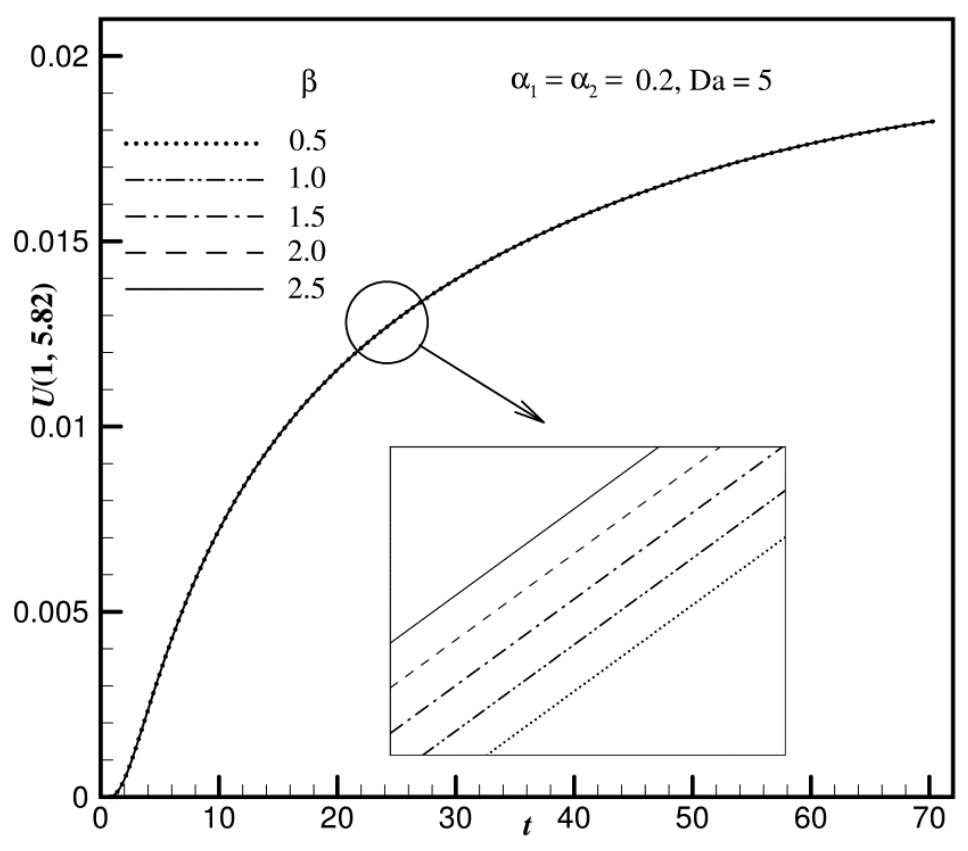

(4a)

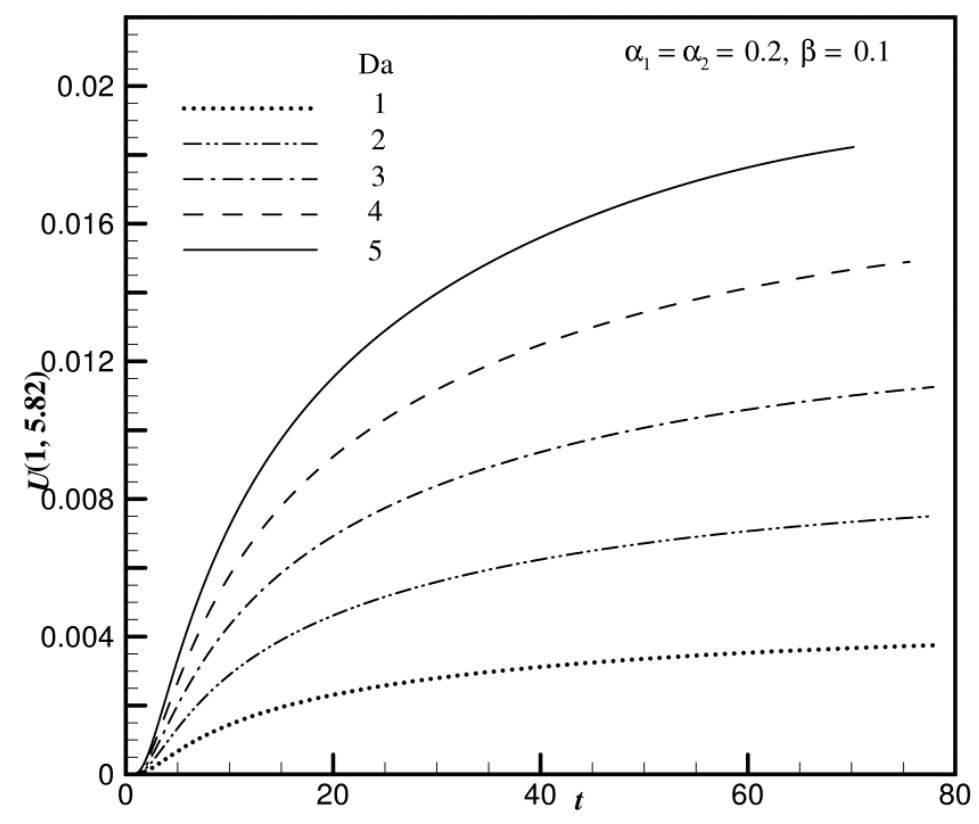

(4b)

Fig. 4. Time-dependent velocity profile $(U)$ versus time $(t)$ at the point $(1,5.82)$ for the effect of (a) $\beta ; \&$ (b) Da. 


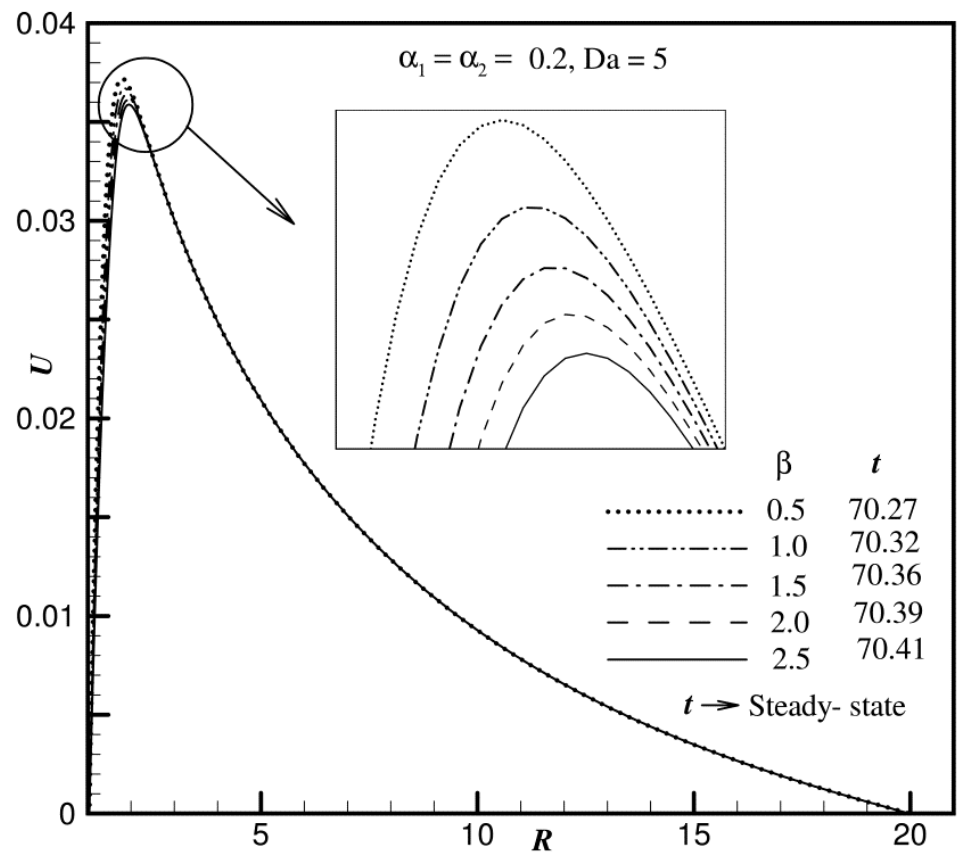

(5a)

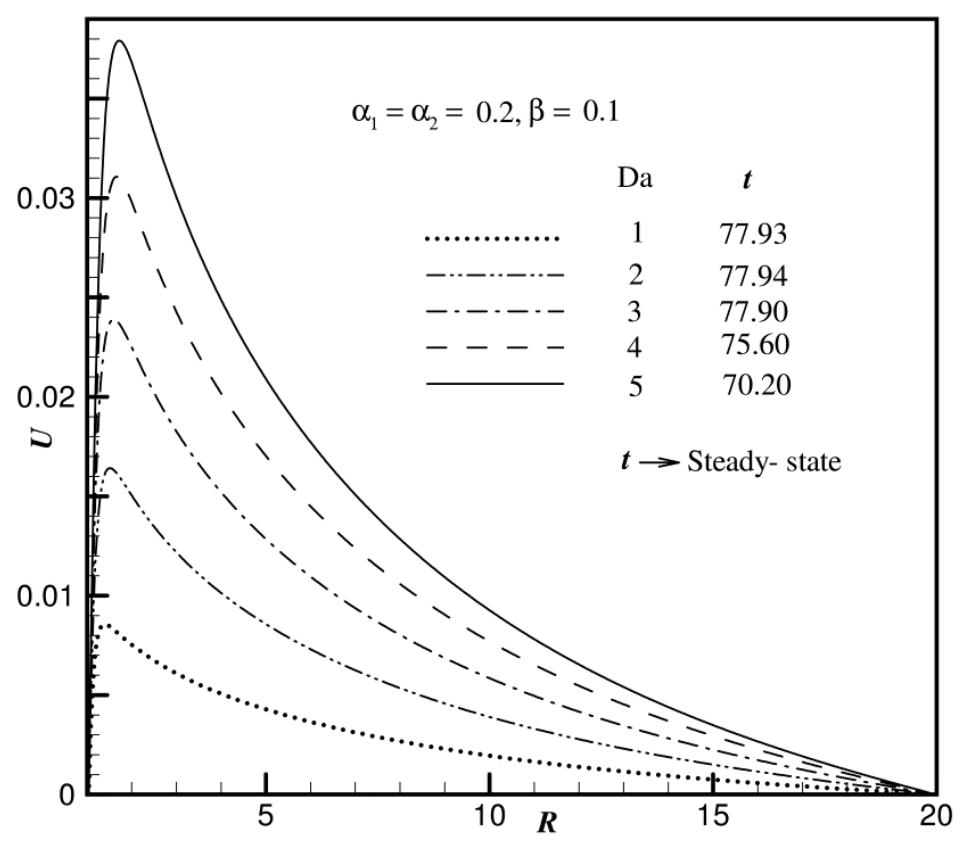

(5b)

Fig. 5. Simulated time-independent state velocity profile $(U)$ versus $R$ at $X=1.0$ for the effect of (a) $\beta ; \&$ (b) Da. 


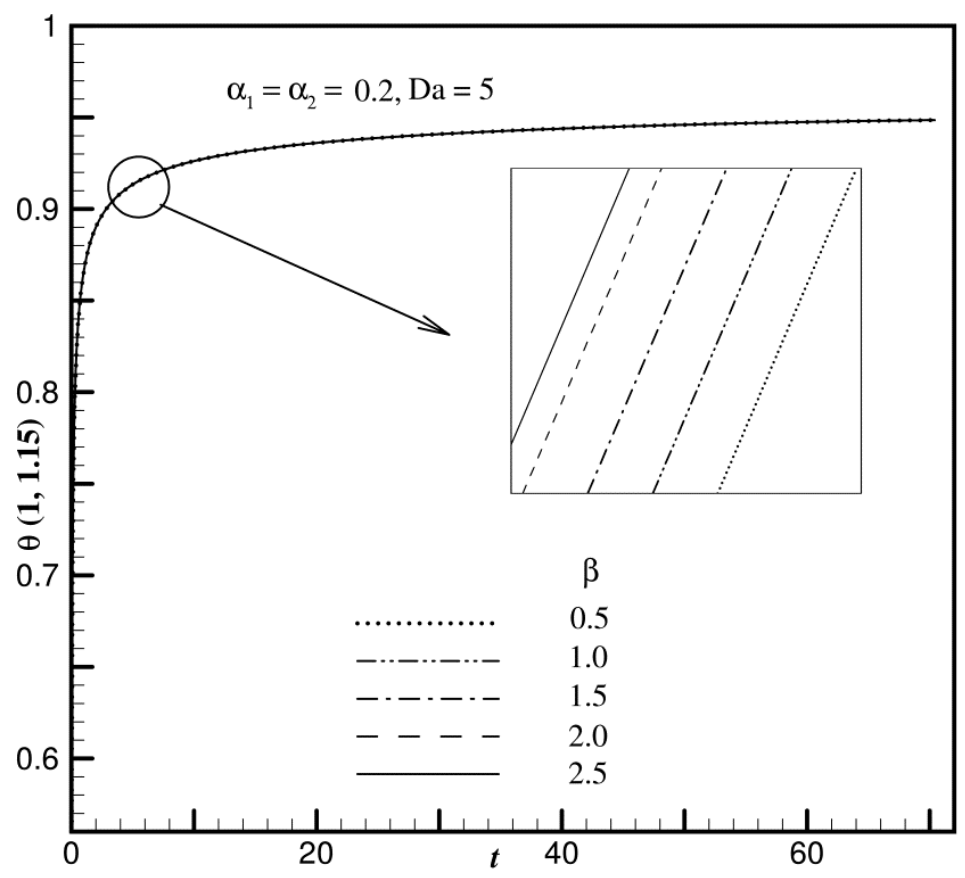

(6a)

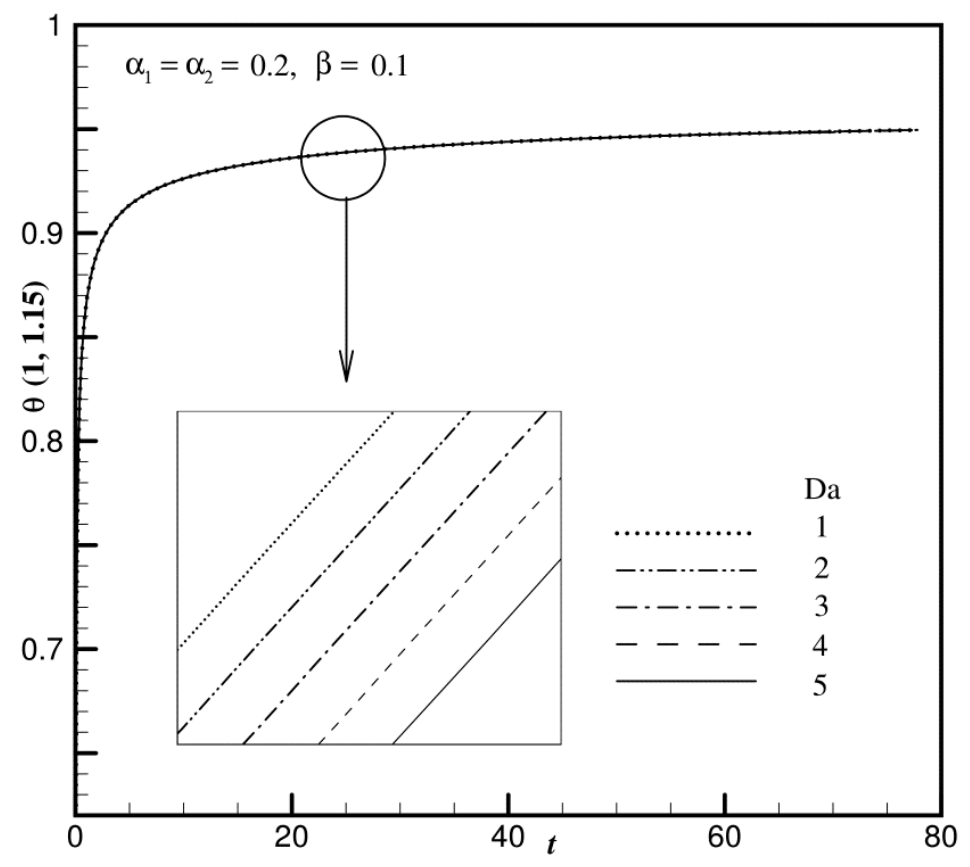

(6b)

Fig. 6. Simulated time-dependent temperature profile $(\theta)$ versus time $(t)$ at the point $(1,1.15)$ for the effect of (a) $\beta ; \&$ (b) Da. 


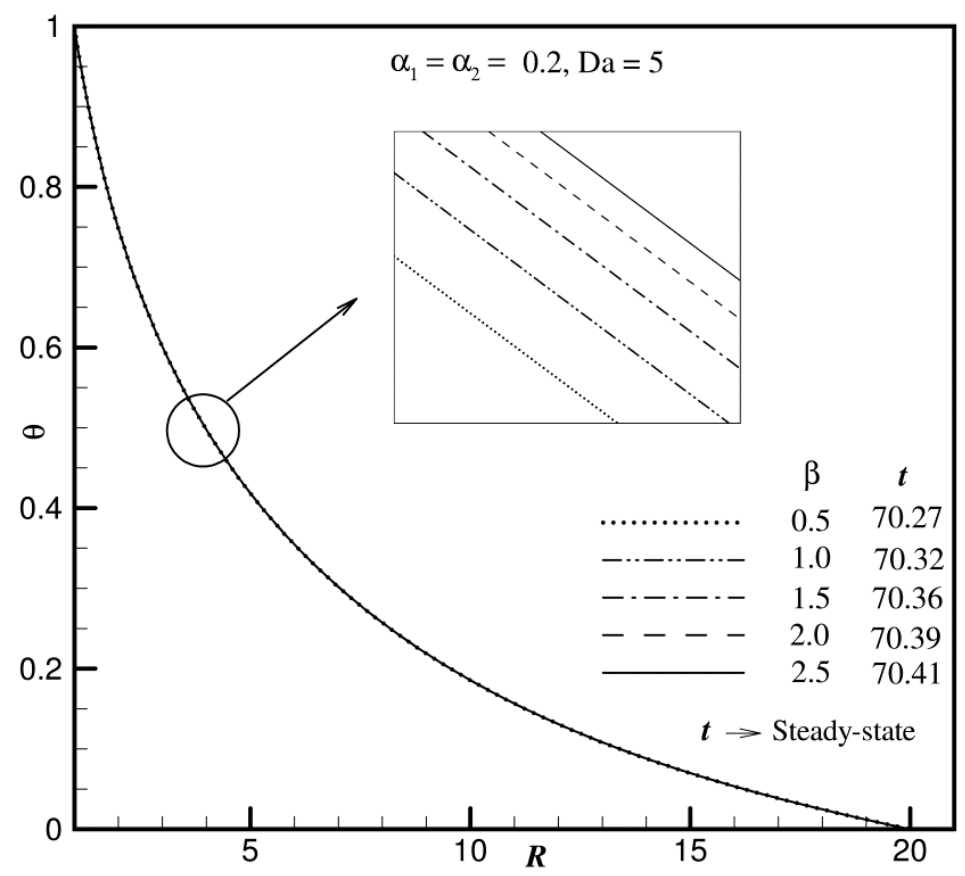

(7a)

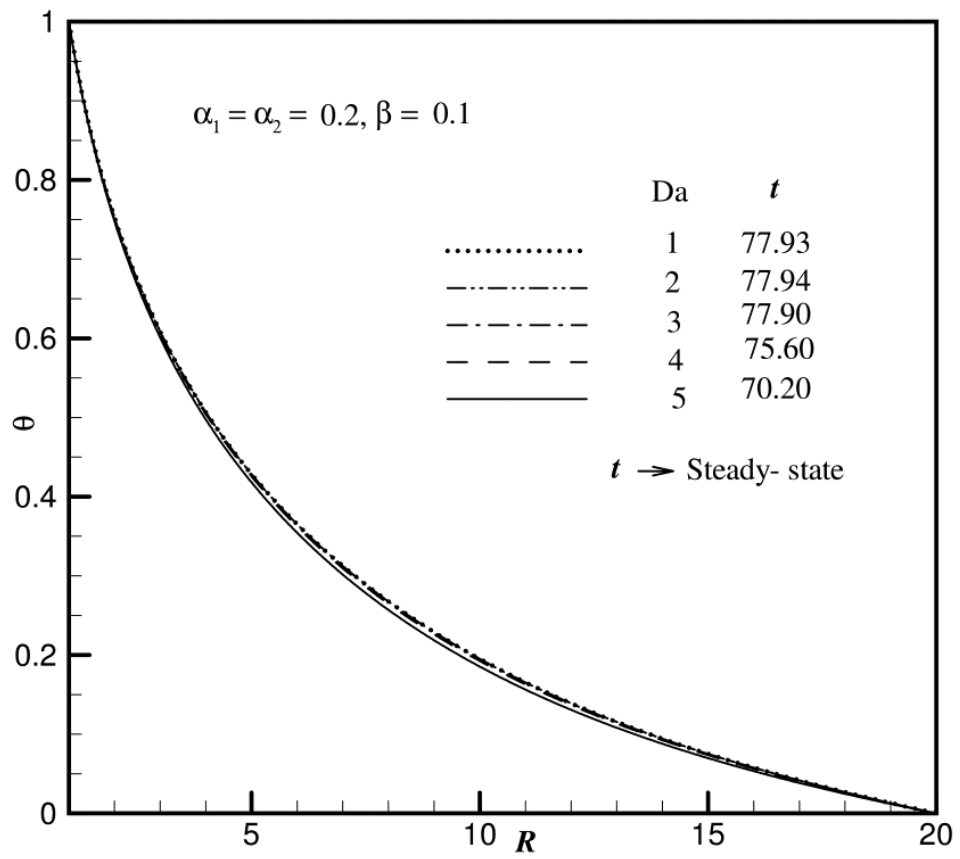

(7b)

Fig. 7. Time-independent state temperature profile ( $\theta$ ) versus $R$ at $X=1.0$ for the effect of (a) $\beta$; $\&$ (b) Da. 


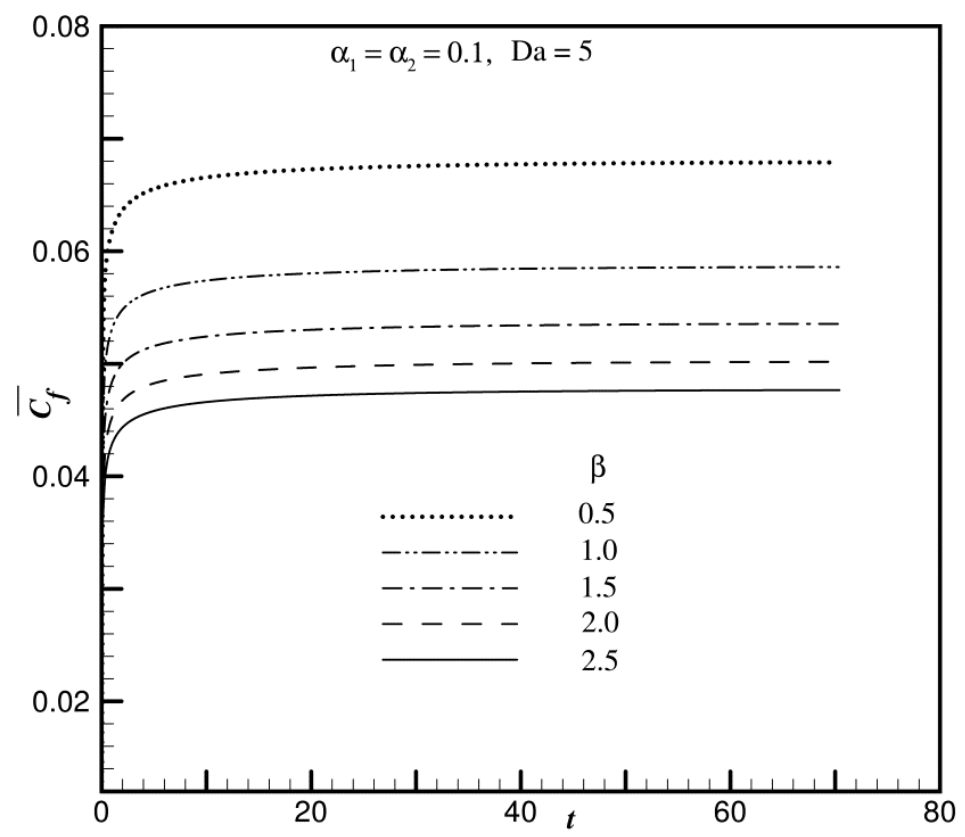

(8a)

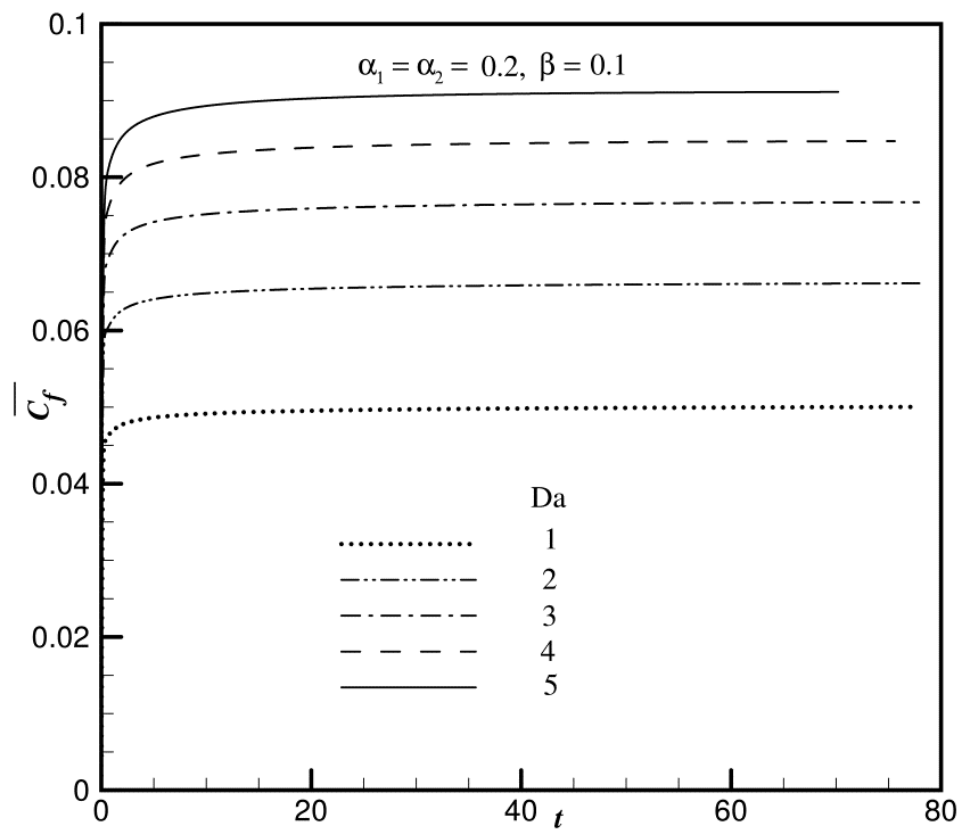

(8b)

Fig. 8. Average momentum transport coefficient $\left(\overline{C_{f}}\right)$ profile against $t$ for the effect of (a) $\beta ; \&$ (b) $\mathrm{Da}$. 


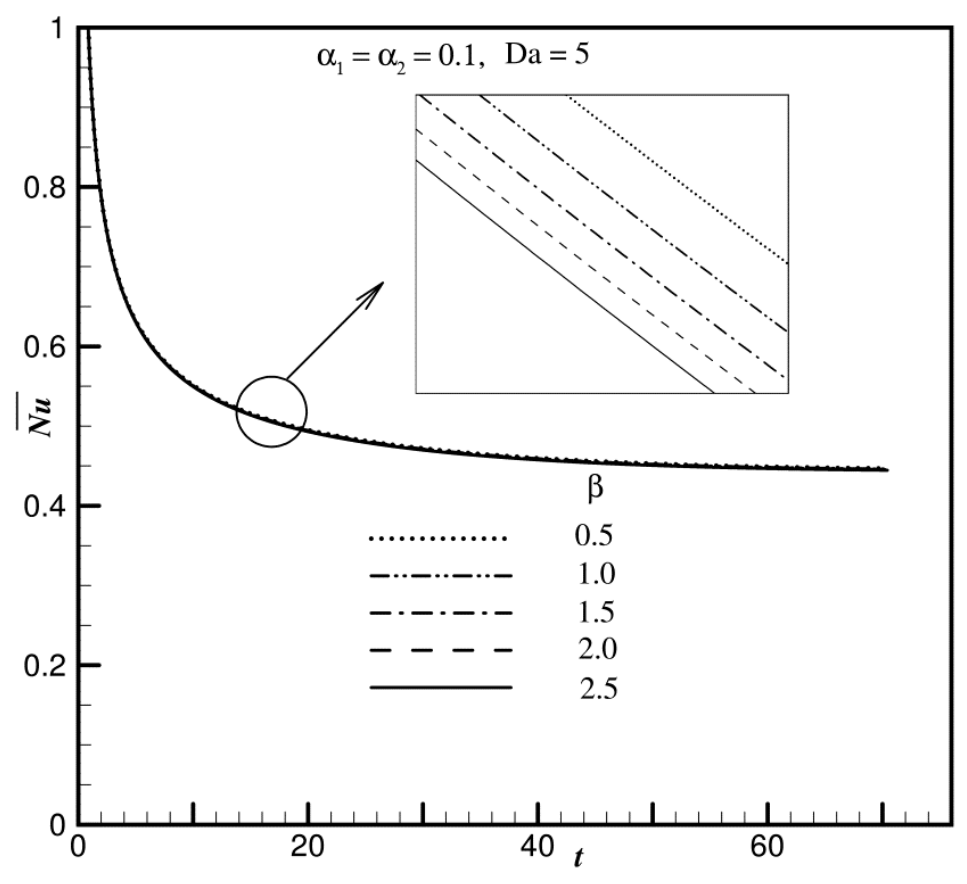

(9a)

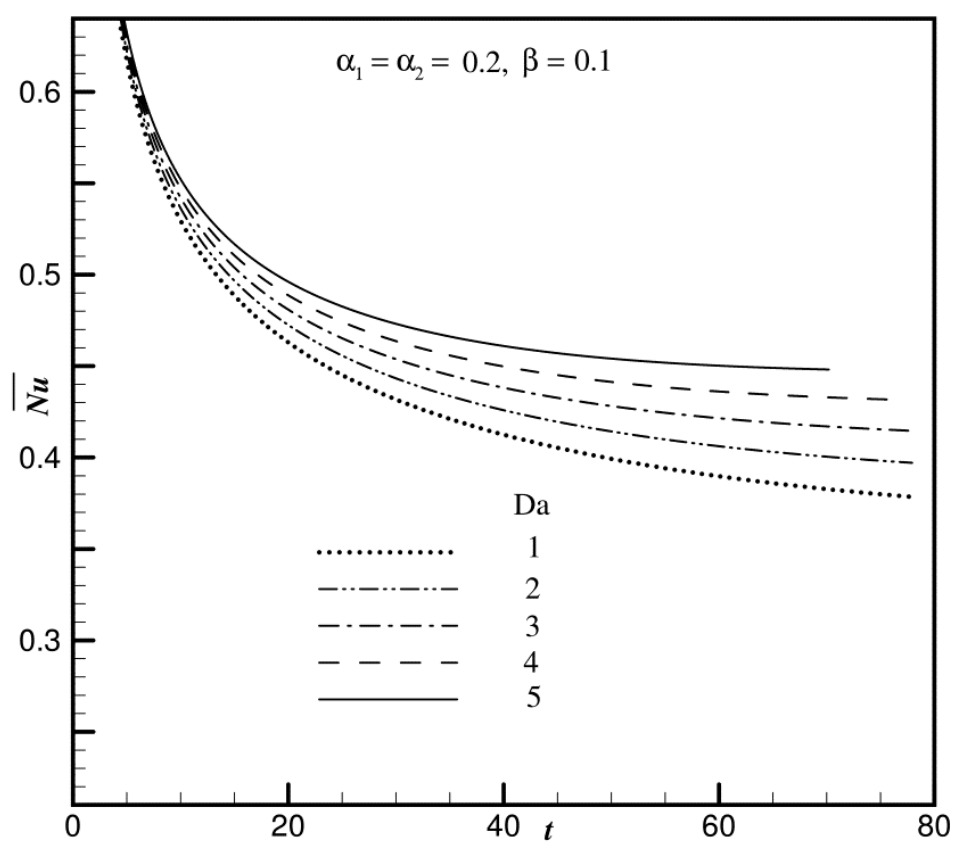

(9b)

Fig. 9. Average heat transport coefficient $(\overline{N u})$ profile against $t$ for the effect of (a) $\beta$; \& (b) Da. 


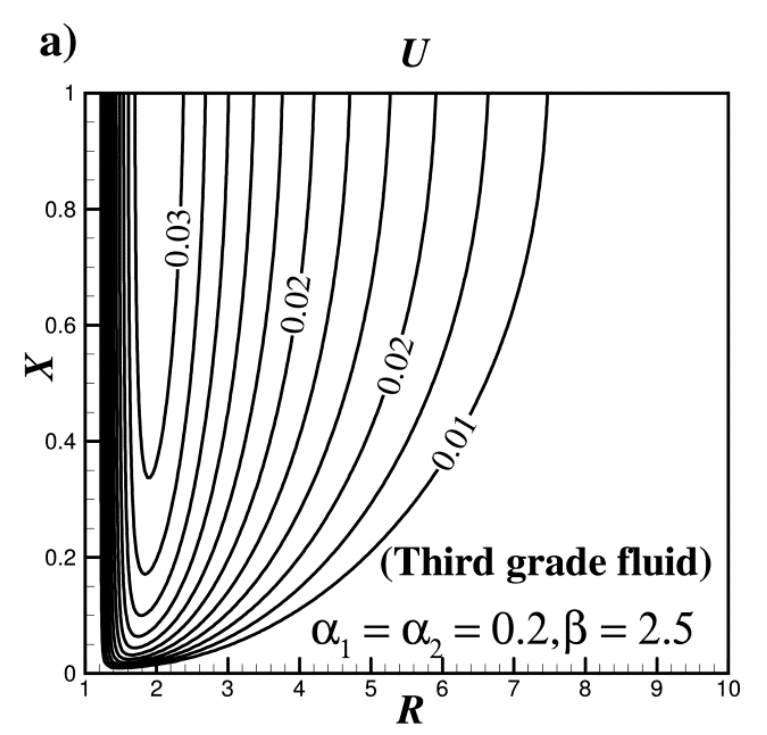

b)

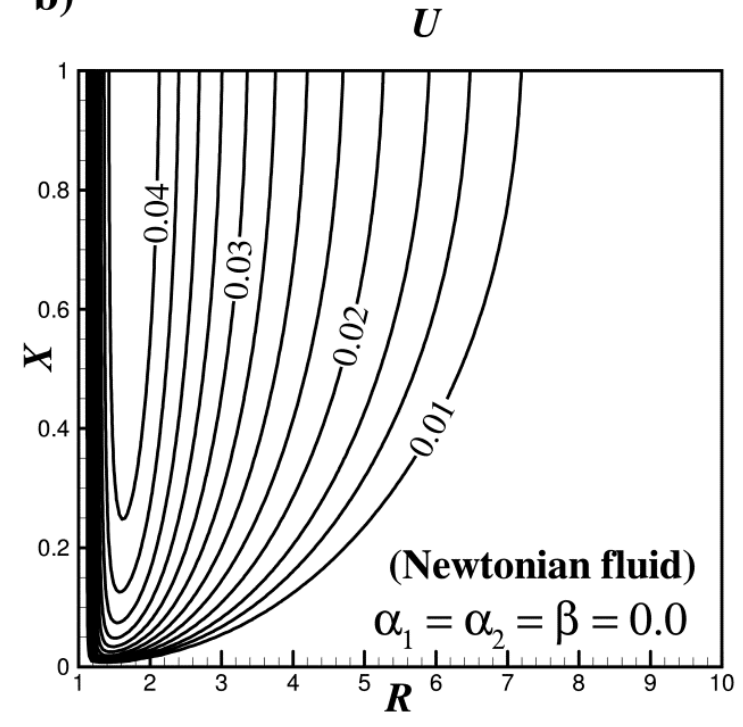

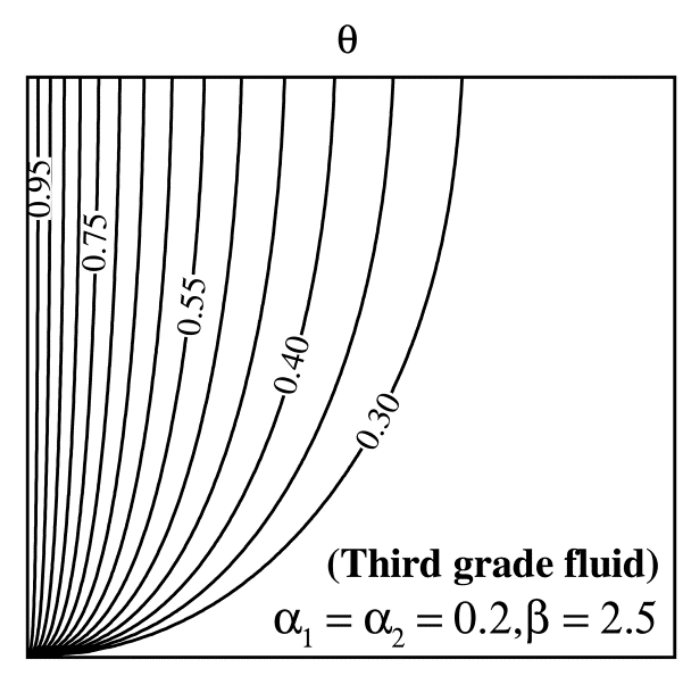

$\theta$

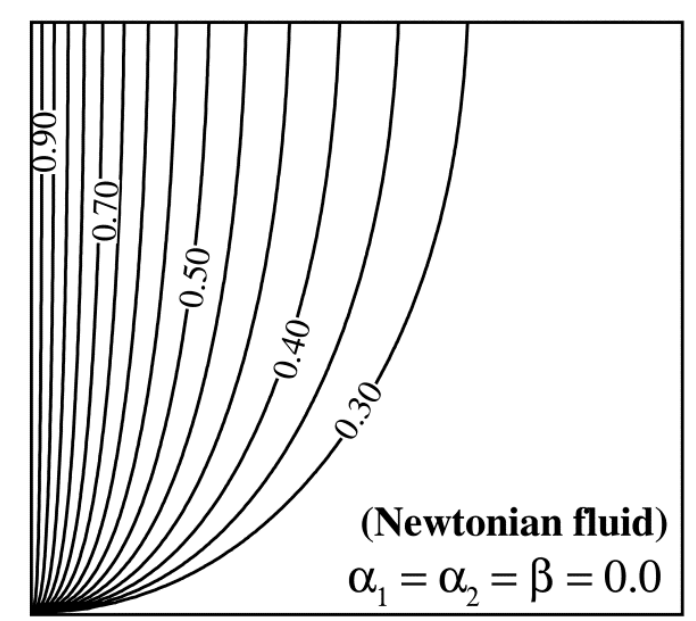

Fig. 10. Time-independent state contours of velocity $(U)$ and temperature $(\theta)$ in $2 \mathrm{D}$ coordinate system $(X, R)$ with fixed values of $P r=0.63$ and $\mathrm{Da}=5$ for (a) Third-grade fluid $\left(\alpha_{1}=\alpha_{2}=0.2\right.$, $\beta=2.5) ; \&$ (b) Newtonian fluid $\left(\alpha_{1}=\alpha_{2}=\beta=0\right)$. 